\title{
II. »Strenae« und »étrennes« - antike Wurzeln, volkskulturelle Traditionen und höfischer Brauch
}

\section{1. "Strenae"}

Die französische Sprache hat mit dem Wort étrennes für die Neujahrsgeschenke einen eigenen Begriff ${ }^{1}$, der zugleich das zentrale Charakteristikum des Überlieferungskörpers ist und den Quellenbestand zu einer quantifizierbaren Größe macht ${ }^{2}$. Etymologie ${ }^{3}$ und Tradition wurzeln in der Antike4: insbesondere die republikanischen und kaiserzeitlichen Römer übten

1 Zu Definitionen lexikalischer Natur siehe u.a. LABORDE, Glossaire, 1872, S. 307f. (Stw. "Estraines« mit weiteren Nachweisen); DuCANGE, Glossarium, Bd. 2, 1883, S. 324 (Stw. "Estrena«); SAINTE-PALAYE, Dictionnaire historique de l'ancien langage françois, Bd. 6, S. 106 (Stw. "Estraigne«); GAY 1887, S. 679-680 (Stw. "Étrennes« im Bd. 1 seines "Glossaire archéologique du moyen âge et de la renaissance«); CHEVALIER, Répertoire des sources historiques du moyen âge. Topo-bibliographie, Bd. 1, 1894, Sp. 1053 (Stw. "Étrennes« mit zahlreichen bibl. Nachweisen); CAIX DE SAINT-AYMOUR, Amédée de: Stw. »Étrennes (hist.)«, in: La Grande Encyclopédie, Bd. 16, S. 694; Dictionnaire historique des arts, métiers et professions, 1906, S. 314 (Stw. »étrennes«, mit der falschen Angabe »c'est le dimanche qui suivait le $1^{\text {er }}$ janvier«, vgl. Ordonnances des Roys de France, ed. SECOUSSE, Bd. 3, 1732, S. 531 die Bestimmung, daß sich die Zunft der Tuchmacher von Rouen am Sonntag nach den estraines zu versammeln habe); GRANDSAIGNES D'HAUTERIVE, Dictionnaire d'ancien français, 1947, S. 270; BAILLY, Paul: Art. »I. Etrennes religieuses«, in: Dictionnaire de spiritualité, Bd. 4, 1960, Sp. 1527-1532, und GREIMAS, Dictionnaire de l'ancien français, 1968, S. 271. Der umfangreichste Artikel findet sich in: Grand dictionnaire universel du XIX ${ }^{e}$ siècle, Bd. 7, 1870, S. 1073-1075, siehe hier v.a. S. 1073-1074 - in Bd. 4 des aktuellen Larousse ist dieser Artikel zusammengeschmolzen auf nicht einmal eine Spalte, siehe dort S. 784f. (Stw. sétrenne«). Die Encyclopédie, hg. von DIDEROT, D'ALEMBERT, 17 Bde., 1751-1780, verzeichnet das Stw. »étrennes« o.ä. überhaupt nicht mehr.

2 Hierzu im vorliegenden Kap. unten Abschn. III.

3 Siehe die Stw. in DuCANGE, Glossarium, Bd. 3, 1884 (Stw. "strena《 S. 611f., Stw. "strenare S. 612) und ebd., Bd. 4, 1885 (Stw. "Kalendae» S. 481-485); TOBLER, LOMMATZSCH, Altfranzösisches Wörterbuch, Bd. 3, 1954 (Stw. »estreine« Sp. 1470-1472, Stw. "estrener« Sp. 1474); MEYER-LÜBKE, Romanisches Etymologisches WÖRTERBUCH, 1968, S. 8296; GAMILLSCHEG, Etymologisches Wörterbuch der französischen Sprache, 1969, S. 402, und DAUZAT, DUBOIS, MITTERAND, Nouveau dictionnaire étymologique, 1971 (Stw. »étrenne« [Sing.!] S. 282). BLOCH, WARTBURG, Dictionnaire étymologique et historique de la langue française, 1932 kennt ein Stw. étrennes o.ä. nicht.

4 Siehe ausgehend von den römisch-antiken Ursprüngen (direkte griechische Entsprechungen der strenae lassen sich nicht nachweisen, vgl. auch FINLEY 1968, hier zu Geschenken S. 56ff., S. 99ff., 129ff.), die Beziehung zwischen étrennes und strenae hervorhebend, die fast durchwegs älteren Arbeiten BARRE 1587; VIGIER 1674 (vgl. SAZERAC DE FORGE 1863); BORDELON 1699; DUSSERT 1786; Origine des étrennes, 1787; S. F. 1814; SPON 1838 (in: LEBER 1838, S. 1-20 - entspr. SPON 1674 -, 20-25 - entspr. SPON 1683. Jacob SPON war ein der Aufklärung verpflichteter Gegner der étrennes, die er als Aberglauben an die Macht des Neujahrstages abtat. Wohl weil die Praxis des Schenkens zum neuen Jahr aber auch höfischer Brauch war, erschienen seine ersten Äußerungen zu diesem 
sich in dem Brauch, sich zum neuen Jahr zu beschenken, und hatten für ihre Gaben den Begriff strenae ${ }^{5}$. Der erste Januar als Tag des Amtsantritts der neuen Konsuln gab mit den sich anschließenden konsularischen Spielen in der ersten Januarwoche und dem Gebot an die Konsuln, sich großzügig zu zeigen,

Thema 1674 und 1683 anonym. Erst die posthum aufgelegte Publikation von 1781 trug seinen Namen, ebenso wie diejenige von 1838 mit kritischen Anmerkungen des Herausgebers. Gegen Jacob SPON siehe René Joseph TOURNEMINE in seiner »Lettre sur les étrennes et sur les dissertations de Spon et de Lipenius«: „Donner pour recevoir est un sentiment fort naturel [...] «: TOURNEMINE 1704, hier S. 26); SAZERAC DE FORGE 1863 (entspr. VIGIER 1674 mit einer bibliographischen Notiz von Adhémar SAZERAC DE FORGE) und MÜLLER 1881, dann das Stw. "Circoncision (fête de la)«, in: CABROL, Dictionnaire, Bd. 3,2, 1914, Sp. 1717-1728, RODGERS 1940 (hier S. 88-92 zur Kontinuität des römischen Neujahrstages im Mittelalter) und SCHMITT, Die Logik der Gesten, 1993, S. 79f. sowie STRATFORD 1995. $\mathrm{Zu}$ den englischen »new year's gifts« und ihrer Tradition BRADY, Clavis Calendaria, Bd. 1, 1815, S. 140ff. (unter dem Abschn. "Circumcision«), vgl. Popular Antiquities of Great Britain, ed. BRAND, HAZLITT, 1870, hier das Kap. »New Year's Day«, S. 6-12, und HolT 1884, hier S. 158.

5 Nur die Neujahrsgeschenke wurden als strenae bezeichnet, andere Festgeschenke hießen sportulae, siehe BAUDY 1987, S. 2 mit Anm. 2 mit weiteren Nachweisen. - Siehe die einschlägigen Lexikaartikel in »Paulys Realencyclopädie der classischen Altertumswissenschaften $\ll$ : NiLSSON, Martin P.: Art. »Kalendae Ianuariae«, in: RE, Bd. 10, 1919, Sp. 1562-1564; Hug, Alfons: Art. »Stips«, in: RE, 2. Reihe, Bd. $3(2,6), 1929$, Sp. 25382540; NilsSON, Martin P.: Art. "Strena«, in: RE, 2. Reihe, 7. Hbd., 1931, Sp. 351-353, und SCHUSTER, Mauriz: Art. »Verbena«, in: RE, 2. Reihe, 15. Hbd., 1955, Sp. 973-976, alsdann die umfangreichen Aufsätze DEUBNER 1912 und FLINCK 1921 (»Auguralia und Verwandtes«, hier »8. Die Strenae und das Augurium Salutis«, S. 50-58), sowie die in diesen Zusammenhang gehörenden Lexikaartikel und Aufsätze HAMILTON-GRIERSON, Philip James: Art. »Gifts (Primitive and Savage)«, in: ERE, Bd. 6, 1913, S. 197-209 (sehr umfangreich mit zahlr. Nachweisen); PEARSON, A. C.: Art. "Gifts (Greek and Roman)«, in: ERE, Bd. 6, 1913, S. 209-213; EDSMAN, C.-M.: Art. »Gabe«, in: RGG, Bd. 2, 1958, Sp. 1183f.; ElLADE, Mircea: Stw. »Neujahr«, in: RGG, Bd. 4, 1960, Sp. 1419f.; HERTZSCH, Erich: Stw. »Neujahrsfest«, in: RGG, Bd. 4, 1960, Sp. 1420f., und STUIBER, Alfred: Art. "Geschenk «, in: RAC, Bd. 10, 1978, S. 685-703. - Dirk SCHLINKERT, vormals TU Darmstadt, jetzt Wolfsburg, ist neben zahlreichen Quellenhinweisen auf Neujahrsgeschenke und -brauchtum in der römischen Antike auch der Hinweis auf das Werk von Hedwig KENNER über »Das Phänomen der verkehrten Welt in der griechisch-römischen Antike« zu verdanken: KENNER 1970, die S. 82-95 das antike Neujahrsfest mit weit in die Frühzeit reichenden Ursprüngen in den Kontext der Saturnalienfeiern einordnet und in diesem Sinn als »Unterdrücktenfeier« definiert (vgl. HEERS 1971, S. 119ff., der die spätmittelalterlichen Narrenfeste als »critique sociale« versteht). Wichtig und grundsätzlich zur »Neujahrsfeier im römischen Kaiserreich « MÜLLER 1909. Auch ältere Arbeiten nahmen sich der strenae an, so Theodorus MARSILIUS (Pseudonym: Claudius MUSAMBERTIUS) unter dem Titel »Historia

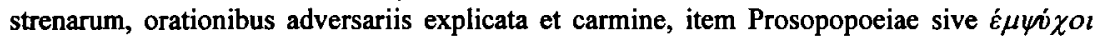

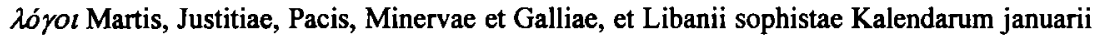
graeca expressio, cum interpretatione et notis«: MARSILIUS 1609, und Martinius LIPENIUS unter der Überschrift »Strenarum historia, a prima origine, per diversas regum, consulum, et imperatorem Romanorum, nec non episcoporum aetates, ad nostra usque tempora«: LIPENIUS 1699. Die einschlägige Monographie zu den Kalendae Ianuariae stammt von Michel MESLIN: MESLIN 1970. Behandlung im Rahmen verwandter Themenbereiche u.a. bei NILSSON 1918 und MiCHELS 1967 (siehe auch S. 51, Anm. 70). 
zusätzlichen Anlaß, den Neujahrstag festlich zu begehen ${ }^{6}$ - wobei die materielle konsularische Großzügigkeit ihren passenden Ausdruck in dem Begriff sparsio fand, was in diesem Zusammenhang "parfümierter Regen« heißen sollte ${ }^{7}$. Die beste Darstellung über das römische Neujahrsbrauchtum, an der sich im folgenden orientiert wurde, stammt von Dorothea Baudy8.

Namensgeberin für die strenae sei die Göttin Strenia gewesen, die «Aufseherin über die Siege«, deren Heiligtum am Anfang der Via Sacra gelegen habe9. Zur Ehre dieser strenuum machenden Göttin ${ }^{10}$, zu verstehen als Attribuierung des dem Jahresbeginn ${ }^{11}$ innewohnenden Wunsches nach glücklichem Neuanfang ${ }^{12}$, habe man, anfangs der Gottheit, dann einander Lorbeer-

6 Vgl. Delmaire 1898, S. 568-575. CAMERON, SCHAUER 1982, S. 141f. berichten in ihrem Aufsatz über »The Last Consul«, wie der byzantinische Kaiser Justinian II. (685-695 und 705-711 n.C.) diesen Brauch wieder aufleben ließ, siehe auch unten S. 43, Anm. 33.

7 Vgl. auch STAROBINSKI 1994, S. 19f.; die Gaben wurden durch einen eigenen dispensator in das Zirkusrund geworfen und zweckmäßiger Weise dann auch missilia genannt. Dieser Brauch wurde noch in Chlodwigs Frankenreich geübt, siehe ebd., S. 27. Eine grundlegende Arbeit zur antiken Einstellung dem Geben gegenüber aus rechtshistorischer Sicht stammt von BRUCK 1954, siehe hier v.a. S. 121ff.: "Die >Liberalitas` der Römer und das Recht der Schenkungen in der republikanischen und klassischen Periode«, 126ff.: »Justinian und die Einführung des Motivs der Selbstlosigkeit in die Digesten«.

8 BAUDY 1987. Der Aufsatz ist mit reichhaltigen Quellenbelegen versehen, die allerdings nur exemplarisch nachgeprüft worden sind.

9 Vgl. ebd., S. 2 mit Anm. 14f. mit weiteren Nachweisen. RADKE 1979, S. 294 führt "Strenia《 in seiner Liste der italischen Götter, hier auch der Hinweis auf den Standort des Heiligtums.

10 Vgl. Baudy 1987, S. 4 mit Anm. 16-22 mit weiteren Nachweisen.

11 Siehe unten S. 51, Anm. 70 und die folgende Anm. 12. Siehe BAUDY 1987, S. 1 mit Anm. 2 mit weiteren Nachweisen. - Ianus, der Namensgeber für den Monat Januar, findet sich in sinnfälliger Entspr. auf zum Jahresbeginn verschenkten (Gedenk-) Münzen wie dem Kupferas, siehe ebd., S. 6 mit Anm. 30, vgl. v.a. NILSSON, Martin P.: Art. „Kalendae Ianuariae«, in: RE, Bd. 10, 1919, Sp. 1562-1564, und MESLIN 1970, hier S. 35, sowie SCHMITT, Heidenspaß und Höllenangst, 1993, S. 79.

12 Vgl. BAUDY 1987, S. 18 f. mit Anm. 75-79 mit weiteren Nachweisen, die S. 18f. mit Anm. 75 und S. 79 - unter Verweis auf die religionsphilosophisch-anthropologischen Ausführungen bei ELIADE 1953 (siehe hier v.a. das zweite Kap. »Die Erneuerung der Zeit«, S. 77-136; vgl. ElIADE 1957 über "Das Heilige und das Profane. Vom Wesen des Religiösen«, hier insbes. S. 40ff.) - das neue Jahr als Zäsur und Termin der Erneuerung für die ganze Gesellschaft sieht, an den sich "mancherlei Sorgen und Hoffnungen knüpfen «, folgerichtig S. 19 dieses Datum in Anlehnung an Arnold van GENNEP zu den writes de passage zählt, siehe ebd., S. 18, Anm. 75, nach der Originalausgabe der »rites de passage« von 1909, S. 254ff. (benutzt in der dt. Übers. GENNEP 1986): "Die Symbolik des Neubeginns hat schließlich in Rom wie anderswo [bspw. Ägypten und China, siehe LAUM 1960, S. 142 mit Anm. 44-47, bspw. Babylon, siehe KUHRT, Amélie: Usurpation, conquest and ceremonial: from Babylon to Persia, in: Rituals of Royalty, hg. von CANNADINE, PRICE, 1987, S. 22-55, hier S. 31-55, J.H.] ihre kosmogonische Dimension. Die Riten des Jahresanfangs swiederholen den Anfang der Welt, den Übergang vom Chaos zum Kosmos«. Siehe zu GENNEP 1986 BERKING 1996, S. 33f. Allerdings, so BERKING ebd., S. 43 richtig in Anlehnung an CHEAL 1988, S. 149f., thematisieren Übergangsriten in erster Linie individuelle Status- 
zweige geschenkt ${ }^{13}$. Diese Lorbeerzweige und, der Jahreszeit angepaßt, kleine Lampen, seien in die Eingangsbereiche der Häuser gehängt worden ${ }^{14}$ und »öffentlich zur Schau gestellt, teilen sie wie in einer Art Soziogramm die Beliebtheit eines Bürgers mit, machen ablesbar, in welchem Ausmaß er in das Geflecht sozialer Beziehungen integriert ist, mit wie vielen anderen er Austauschbeziehungen pflegt. «15 Weitere, später dann aus dem genuin sakralen Bereich herausgelöste, übliche römische Neujahrsgeschenke waren Datteln, Feigen und Honig, vergoldete Datteln, Münzen und schließlich neben den schon erwähnten Lämpchen tönerne Sparbüchsen mit der eingravierten Glückwunschformel annum novum faustum felicem (tibi) ${ }^{16}$.

Insbesondere das Verschenken von Geld provozierte die zeitgenössische Kritik und nahm zum Teil Argumente gegen den Gabentausch zum neuen Jahr von christlich-kirchlicher Seite vorweg: die strena sei ein sacrum munus, und ein heiliges Geschenk könne nicht schlicht aus Geld bestehen ${ }^{17}$. Da eine Gabe eine Gegengabe verlange, könne ein Geldgeschenk nur mit einem Geldgeschenk erwidert werden, wodurch das Brauchtum zum neuen Jahr zum Geschäft verkomme, klagte Sueton in seiner Vita des Kaisers Tiberius und belegte diesen Vorgang mit dem Begriff strenarum commercium ${ }^{18}$. Tatsächlich mündete der Austausch von Geldgeschenken an den Kalenden des Januar aber in eine Art Sondersteuer ${ }^{19}$. Augustus noch hatte den ihm dargebrachten strenae in Geldform eine die Einzelgabe jeweils übersteigende Gegengabe hinzugefügt und den Gesamtbetrag in eine Stiftung zur Errichtung

änderungen, der Gabentausch zum neuen Jahr teile aber (obwohl nicht ausdrücklich erwähnt, aber unter »kalendarisch wiederkehrende Ereignisse « zu subsumieren) mit diesen wegen des Datums zwar das Thema des Wandels in der Zeit, betone aber als »Progressionsritus« Dauer und Kontinuität von Beziehungen.

13 Vgl. BAUDY 1987, S. 3 mit Anm. 13, und S. 5 mit Anm. 23-28.

14 Vgl. ebd., S. 20ff.

15 Ebd., S. 23. - Dorothea BAUDY streift auch die verhaltenswissenschaftliche Auffassung, $\mathrm{da} ß$ es sich beim Gabentausch im Grunde um ritualisiertes Brutpflegeverhalten handeln könne (S. 15, Anm. 61), aufgehängt an dem Beispiel, daß Vögel einander Nestbaumaterial (Zweige!) überreichen, siehe EIBL-EIBESFELDT 1984, S. $128 \mathrm{ff}$. (das Geschenk als ritualisierte Fütterung), vgl. BERKING 1996, S. 119. Vgl. auch SAUTER 1969, S. 16, der berichtet, wie im mittelalterlichen Europa die jungen Burschen ihre Mädchen zum Jahresanfang neben Äpfeln und anderem Obst mit Mistelzweigen beglückten. Daß sich hier tatsächlich auch ein biogenetischer Determinismus offenbart, siehe auch oben S. 12, Anm. 16, darf bezweifelt werden.

16 Vgl. Baudy 1987, S. 2 mit Anm. 5-7 mit weiteren Nachweisen Zu den Sparbüchsen v.a. GRAEVEN 1901.

17 Siehe BAUDY 1987, S. 3 mit Anm. 10 f.

18 Siehe ebd., S. 7f. mit Anm. 33.

19 Siehe BAUDY 1987, S. 8 mit Anm. 37 - Vgl. SAUTER 1969, der S. 16 berichtet, daß Heinrich III. von England die Londoner im 13. Jh. zu einer Neujahrsabgabe zwang. Über die Tatsache selbst, daß der Brauch, einander Neujahrsgeschenke zu überreichen, in London geübt wurde, informiert bspw. "Sir Richard Whittington. Lord Mayor of London [1397, J.H.] «, ed. BESANT, RICE 1881, hier S. 96. 
von Standbildern eingebracht 20 . Tiberius pflegte anfangs mit dem vierfachen Gegenwert auf die ihm überreichten Geschenke zu antworten, was zu einer über den Jahresanfang hinaus sich ausdehnenden Schenkpraxis führte, so daß der Kaiser zunächst versuchte, den Brauch wieder auf den ersten Januar zu beschränken, ihn anschließend dann aber ganz verbot ${ }^{21}$. Caligula hingegen tat öffentlich kund, er nehme strenae an, ohne aber tatsächlich daran zu denken, sich zu revanchieren und der Codex Theodosianus legte schließlich fest, wie hoch der Geldbetrag zu sein habe 22 .

Einer der ersten christlichen Kritiker des Austausches von strenae war Augustinus, der sich vor allem gegen das diesem Austausch immante Prinzip des sich auf der materiellen Ebene äußernden Zusammenhangs von Gabe und Gegengabe wandte und den strenae den christlichen Almosengedanken gegenüberstellte: Dant illi strenas, date vos eleemosynas ${ }^{23}$. Spätere synodale und konziliare Beschlüsse geben dann Aufschluß darüber, daß es ein populäres - Neujahrsbrauchtum auf gallischem Boden nicht nur im Rahmen der römisch-antiken Kultur gegeben hat ${ }^{24}$, sondern auch, daß dieses Brauchtum sich offensichtlich trotz kirchlicher Bestrebungen wie der Einrichtung des Festes der Circumcision ${ }^{25}$ als Fest der Beschneidung Christi zur Verstärkung der zu feiernden Oktav Christi Geburt nicht hat unterbinden lassen $^{26}$.

Siehe BAUDY 1987, S. 8 mit Anm. 35f.

Siehe ebd., S. 7f. mit Anm. 33f.

22 Siehe ebd., S. 8 mit Anm. 36.

23 Zitiert nach ebd., S. 8. Vgl. SCHMITT, Heidenspaß und Höllenangst, 1993, S. 79f. zum argumentativen Hintergrund.

24 Vgl. den keltischen Brauch des "Aguilanneuf«, dazu LABORDE, Glossaire, 1872, S. 123 (zur Übung dieses Brauches im Anjou CLÉMENT-HÉBERY 1846, S. 447), vgl. GENNEP 1988, S. 3473-3482 und DAVIS 2000, S. 38f.

25 Belegt seit 546, siehe KÜSTER 1985, S. 132, anfangs als Bußtag, seit dem 13./14. Jh. als eigentliches kirchliches Fest, siehe v.a. NICKEL, Die heiligen Zeiten und Feste, Bd. 1, 1836, S. 253, der erste Spuren des Beschneidungsfestes in einer Rede des Zeno, Bischof von Verona im 4. Jh., festgestellt hat, und als weitere Hinweise das »Mozarabische Kalendarium«, das »Kalendarium Fronto«, das »Gelasianische Sakrament« und das »Martyrologium des heiligen Hieronymus« nennt. Vgl. DÜRIG, Walter: Art. »Neujahr«, hier »III. Liturgisch«, in: Lexikon für Theologie und Kirche, hg. von HÖFER, RAHNER, Bd. 7, 1962, der Sp. $910 \mathrm{f}$. ausfuhrt, daß im gallischen Liturgiebereich seit dem 6. Jh. die Feier der Circumcisio vorherrschte, in Rom aber erst seit dem 10. Jh., vgl. SCHMITT, Heidenspaß und Höllenangst, 1993, S. 81. Siehe v.a. BÜNGER 1910, S. 12-31 aus kirchlicher Perspektive zum "Busstag als Gegensatz gegen die heidnische Kalendenfeier«, 32-77 „Das Beschneidungsfest« mit zahlreichen Nachweisen. Eine bildliche Umsetzung der Circumcision findet sich bei GERMAIN 1909, S. 106, siehe auch ACO B 302, fol. 27, ed. DEHAISNES, Documents et extraits, Bd. 2, 1886, S. 876: tableau d'ivoire a ymaiges de la Nativité et Circuncision [!] Nostre Seigneur. Erst seit 1969 ist der erste Januar das Hochfest der Gottesmutter Maria. Zur Verbindung von erstem Januar und Marienverehrung ebenfalls BÜNGER 1910, S. 78-85.

26 Zur kirchlichen Neujahrsfeier und ihrer Geschichte v.a. NICKEL, Die heiligen Zeiten und Feste, Bd. 1, 1836 (hier S. 251-256 „Das Fest der Beschneidung Christ, und das neue 


\section{2. "La fête des fous"}

Im Jahr 567 bestimmte die Synode von Tours in ihrem 18. und 23. Kanon: "Von Weihnachten bis Epiphanie ist täglich prandium, weil alle Tage Feste sind. Ausgenommen sind nur die drei Tage im Anfange Januars, auf welche die Väter, um die heidnischen Gewohnheiten zu bekämpfen, Privatlitaneien angeordnet haben. Am ersten Januar, dem Beschneidungsfeste, soll die Messe um $8 \mathrm{Uhr}$ gesungen werden. [...] Einige halten noch den alten Irrthum fest, daß sie den ersten Januar ehren. Andere bringen an Petri Stuhlfeier den Todten Speiseopfer dar, und genießen Speisen, die dem Dämon geweiht sind. Andere ehren gewisse Felsen oder Bäume oder Quellen etc. Die Priester sollen diesen heidnischen Aberglauben ausrotten. ${ }^{27}$

Etwa zehn Jahre28 nach dieser Synode setzte das Konzil von Auxerre fest: »Niemand darf am 1. Januar nach heidnischer Art sich in Kühe (oder alte Weiber) und Hirsche verkleiden oder diabolische Neujahrsgeschenke machen; vielmehr sollen an diesem Tag keine andern Geschenke als sonst verteilt werden. $\ll^{29}$

Jahr«); KLEINERT 1900; BÜNGER 1910; KELLNER 1911; SCHNEIDER 1920/1921; SCHRÖER 1994 und STOLZ 1994. Zum Begriff des Kirchenfestes allg. JUNGMANN 1958.

27 In der dt. Übers. nach HEFELE, Conciliengeschichte, Bd. 3, 1877, S. 22-28, hier S. $25 f$. Siehe BAILLY, Paul: Art. »I. Étrennes religieuses«, in: Dictionnaire de spiritualité, Bd. 4, 1960, Sp. 1527-1532, hier Sp. 1528, mit Nennung der wichtigsten Literatur. Vgl. HAUCK, Kirchengeschichte Deutschlands, Bd. 1, 1887, S. 118 und SCHMITT, Heidenspaß und Höllenangst, 1993, S. 81f. Die genannte Synode wird behandelt bei Odette Pontal im Rahmen ihrer Arbeit über die "Synoden im Merowingerreich«: PONTAL 1986, hier S. 128-135, zu den oben genannten canones ebd., S. 132f. mit zahlreichen weiteren bibl. Nachweisen in den Anm. $41 f$.

28 Datierung unsicher; nach MASSEN 1893 (= MGH LL Conc. 1-1893), S. 178 wie auch PONTAL 1986, S. 167 zwischen 573 und 603, beide orientiert am Episkopat des Aunacharius (Aunarius), wobei PONTAL aber für eine Datierung nach 585 eintritt, weil dieses Konzil auf canones von Macon 585 zurückgreife.

29 In der dt. Übers. nach HEFELE, Conciliengeschichte, Bd. 3, 1877, S. 42-47, hier S. 42. Ed. MASSEN 1893 ( $=$ MGH LL Conc. I-1893), S. 178-184: Concilium Autissiodorense (ebenso PL, Bd. 72, 1849, Sp. 761-768), hier S. 178: Non licet kalendis Ianuarii vetolo aut cervolo facere vel streneas diabolicas observare, sed in ipsa die sic omnia beneficia tribuantur. Vgl. PL, Bd. 57, 1847, Sp. 253-258, 43-48: Homilia XVI. De calendis Januariis, hier Sp. 255f., und ebd., Bd. 94, 1850, Sp. 53-58: Homilia X. In die festo circumcensionis domini, hier Sp. 55f.: Zeitliche, kirchliche und religiöse Aspekte des Neujahrstages, angesprochen in den hier zitierten sermones, denen die Lesungen des temporale im Pariser Brevier entnommen sind, vgl. STRATFORD, J. 1995, S. 44 mit S. 50, Anm. 23. Begriffserklärungen: DUCANGE, Glossarium, Bd. 2, 1883, S. 277f. ("Cervulak) und ebd., Bd. 8, 1887, S. 297f. ("Vetula $)$, vgl. SCHMITT, Heidenspaß und Höllenangst, 1993, S. 80. ZEDLER, Großes vollständiges Universal-Lexikon aller Wissenschaften und Künste, Bd. 23/24, 1740, Sp. 212f. findet unter dem Stw. "Neu-Jahrs-Geschenke» v.a. dieses Verbot von Neujahrsgeschenken erwähnenswert. Zum Konzil von Auxerre FRIEDBERG 1868, S. 64; HAUCK, Kirchengeschichte Deutschlands, Bd. 1, 1887, S. 118; DUCHESNE, Fastes épiscopaux, Bd. 2, 1910, S. 435ff. (hier v.a. S. 440, 446 zu Bischof Aunarius/Aunacharius); CABROL, Fernand: 
Damit wandte sich die Kirche neben dem Gabentausch auch gegen andere Ausprägungen des Neujahrsbrauchtums wie das Maskieren als "frevelhafte Verkehrung der göttlichen Schöpfungsordnung « ${ }^{30}$ und das ausgelassene Feiern $^{31}$, gegen dessen Verbindung mit dem Genuß von Tafelfreuden sie das Gebot des Fastens setzte ${ }^{32}$. Gerade der niedere Klerus scheint dieses in der Tradition der römischen Saturnalien stehende $\mathrm{Fest}^{33}$, das am Ende einer ganzen Reihe ähnlicher Feste besonders seit dem Weihnachtsfest lag (ein Zeitraum, der sich ausdehnen konnte bis zum sechsten Januar und mancherorts schon Anfang Dezember begann), am intensivsten betrieben zu haben ${ }^{34}$. Der Klerus und das niedere (Stadt-)Volk begingen den Neujahrstag in den Kathedralen ihrer Städte als festum asinorum ${ }^{35}$ (Eselsfest), einer besonderen Form des festum stultorum (Narrenfest) beziehungsweise der fête des fous ${ }^{36}$,

Stw. »Circoncision (fête de la)«, in: CABROL, Dictionnaire, Bd. 3,2, 1914, Sp. 1717-1728, hier Sp. 1720; CHARTRAIRE, E.: Art. »Auxerre«, in: Dictionnaire d'histoire et de géographie ecclésiastiques, hg. von BAUDRILlART, VOGT, ROUZIÈS, Bd. 4, 1930, Sp. 939-958 (hier Sp. 945 eine Liste der Bischöfe); KARLE, Josef Anton: Stw. "Konzil«, in: HdA, Bd. 5, 1932/1933, Sp. 191-201, hier v.a. Sp. 194, 199 mit Anm. 39; BACKMUND, Norbert: Art. "Auxerre", in: Lexikon für Theologie und Kirche, hg. von HÖFER, RAHNER, Bd. 1, 1957, Sp. 1138f.; RAHNER, Hugo: Art. "Aunarius«, in: Ebd., Sp. 1106 (zu dem maßgeblichen Bischof Aunarius/Aunacharius, der die 45 canones zu den kirchlichen Gebräuchen der Zeit aufstellte) und BAILLY, Paul: Art. "I. Etrennes religieuses", in: Dictionnaire de spiritualité, Bd. 4, 1960, Sp. 1527-1532 mit Nennung der wichtigsten Literatur.

30 Vgl. SCHMITT, Heidenspaß und Höllenangst, 1993, S. 80f. Möglicherweise war die Verschenkung von phantasievoll geschmückten, anspielungsreich verzierten und farbenprächtigen houppelandes zum Neujahrstag eine höfische Reminiszenz an alte volkskulturelle Verkleidungsbräuche, vgl. STRATFORD 1995, S. 44; siehe auch unten S. 49, Anm. 65 bzw. S. 176, Anm. 271.

31 Vgl. SCHMITT, Heidenspaß und Höllenangst, 1993, S. 80f.

32 Vgl. NiLSSON 1914, S. 60f.

33 Zur "verkehrten Weltu dieser Feiern KENNER 1970, siehe auch oben S. 38, Anm. 5. Auch für Konstantinopel ist ein Weiterleben dieser Traditionen belegt, auch hier ist es von der Kirche verurteilt worden, siehe TINNEFELD 1995, S. 140 mit weiteren Nachweisen.

34 Was bis zur Androhung der Exkommunikation führte, niedergelegt bspw. in den Statuten der Kirche von Nevers 1246, siehe Thesaurus novus anecdotorum, ed. MARTĖNE, DuRAND, Bd. 4, 1717, S. 1069ff. Vgl. DuCANGE, Glossarium, Bd. 4, 1885, Stw. "Kalendae«, S. 481-485, hier S. 483. - Siehe insbes. KINDERMANN, Udo: Art. »Klerikerfeste«, in: LexMA, Bd. 5, 1991, Sp. 1206, und, auf Paris bezogen, PERDRIZET 1933, hier S. 70.

35 Siehe insbes. HEERS 1983, S. 156-164. Ein Beispiel für Rouen und Beauvais bei CLÉMENT-HÉBERY 1846, S. 398-405.

36 Um den damit verbundenen ausgelassenen Festlichkeiten entgegenzutreten, habe die Kirche bereits vor Einführung der Feier Christi Beschneidung den Neujahrstag als Bußtag proklamiert und möglicherweise Messen $a b$ idolis prohibendum abgehalten, zugeschrieben Papst Gelasius (492-496), so DÜRIG, Walter: Art. »Neujahr«, hier »III. Liturgisch«, in: Lexikon für Theologie und Kirche, hg. von HÖFER, RAHNER, Bd. 7, 1962, Sp. 910 f. - Siehe Inventaires mobiliers, hg. von PROST, PROST, Bd. 1, 1902-1904, S. 89, Nr. 584 mit Anm. 5 und zahlreichen weiteren Nachweien. Zu den Narrenfesten siehe ansonsten BÜNGER 1910, S. 86-102, hier nur zu illustrativen Zwecken zitiert, v.a. aber HEERS 1983, hier Kap. III »Das Narrenfest«, S. 123-215, alsdann insbes. TLLLIOT 1751. Siehe DAVIS 1987, S. 106-135 
mit der Wahl von Knabenbischöfen oder Narrenpäpsten, begleitet von Saufgelagen und wilden Tänzen, denen sich maskierte Umzüge anschlossen ${ }^{37}$; eine »kurzlebige Revanche aller Subalternen ${ }^{38}$, vor allem in den deutschen Ländern, Nordfrankreich und England ${ }^{39}$.

Kirchliche Versuche, gegen dieses Brauchtum vorzugehen, lassen sich nicht nur für das frühe Mittelalter nachweisen ${ }^{40}$. Das Basler Konzil $1435^{41}$ und eine epistola contra festum fatuorum der Pariser Universität von $1445^{42}$, die explizit verbot, Narrenbischöfe zu wählen, bestätigen die offensichtliche Vergeblichkeit, das Neujahrsbrauchtum zu unterbinden. Karl VII. hatte, ebenfalls 1445, gar das ganze Fest mit einem Verbot belegt ${ }^{43}$, ebenso Karl der Kühne $1469^{44}$. Noch Philipp der Kühne hatte zum Neujahrstag 1386 die Souveränität besessen, in Tournai einen Bischof der Narren, qui [...] vint devant $\mathrm{Mgr}$ [...] avec autres personnaiges pour l'esbatement de $\mathrm{Mgr}$, mit $6 \mathrm{fr}$. zu beschenken ${ }^{45}$.

Die Kirche wandte sich also vor allem gegen die volkskulturellen Ausprägungen des Neujahrsbrauchtums ${ }^{46}$, hier wiederum in erster Linie gegen diejenigen, die in ihren eigenen Räumen stattfanden ${ }^{47}$. Die höfische Welt

(»Die Narrenherrschaft«) zum frühneuzeitlichen Nachleben und DAVIS 2000, S. $39 \mathrm{mit}$ S. 234, Anm. 7.

37 Hierzu v.a. HEERS 1983, S. 189ff. (»Das verkehrte Fest: der Knabenbischof«). Vgl. LEFÉBURE 1902.

38 HEERS 1983, S. 202.

39 Zum nachmittelalterlichen Neujahrsbrauchtum in der Volkskultur der deutsch-sprachigen Länder SARTORI 1914 und SARTORI, Paul: Art. »Neujahr (1. Januar)«, in: HdA, Bd. 4, 1934/1935, Sp. 1020-1045.

40 Die beste Zusammenstellung bei SCHNEIDER 1920/1921, vgl. NICKEL, Die heiligen Zeiten und Feste, Bd. 1, 1836, S. 251, der Belege bis ins 8. Jh. nennt.

41 Vgl. HelmRATH 1987, S. 335f. mit Anm. 32f.

42 Chartularium Universitatis Parisiensis, ed. DENIFLE, CHÂTELAIN, Bd. 4, 1897, S. 652656, Nr. 2595: Epistola et 14 conclusiones facultatis theologiae Paris. ad ecclesiarum praelatos contra festum fatuorum in Octavis Nativitatis Domini vel prima Januarii in quibusdam ecclesiis celebratum mit weiteren bibl. Hinweisen S. 656.

43 Thesaurus novus anecdotorum, ed. MARTĖNE, DURAND, Bd. 1, 1717, S. 1804, vgl. DUCANGE, Glossarium, Bd. 4, 1885: Stw. »Kalendae«, S. 481-485, hier S. 483f.

44 Siehe PARAVICINI 1999, S. 343f. bezogen auf $\S 153$ der Hofordnung von 1469 Karls des Kühnen.

45 ACO B 1462, fol. 99v, ed. Inventaires mobiliers, hg. von PROST, PROST, Bd. 2, 19081913, S. 212, Nr. 1356. Siehe Anhang - Katalog/Jahreslisten, Nr. 168. Die ebenfalls in diesen Zusammenhang gehörende »Fête des Innocents« in Tournai behandelt CLÉMENT-HÉBERY 1846, S. 61-76.

46 Vgl. die knappen Ausführungen bei VERDON 1980, S. 28-32. Arnold van GENNEP beschreibt ausführlich das (heute noch) vorhandene Brauchtum der Jahreswende: GENNEP 1958 (hier Bd. 1, VII, $1^{\text {er }}$ partie: »Cycle des douze jours «) und wieder GENNEP 1988 (hier Bd. 1, VIII: "Cycle des douze jours. De Noël aux rois«, hier zum »Jour de l'an« S. 3471-3529). Vgl. COREMANS 1844, S. 75 zum Brauchtum im »alten Belgien«.

47 Zur offiziellen kirchlichen Neujahrsfeier der Zeit um 1400 DYKMANs, Le cérémonial papal, Bd. 3, 1983, pass. 
selbst ist offenbar weder angesprochen worden, noch schien sie sich angesprochen gefühlt zu haben ${ }^{48}$. Immerhin waren, was zumindest den Austausch von Geschenken um 1400 anbelangt, hohe Kleriker wichtige Teilnehmer des höfischen Geschenkverkehrs ${ }^{49}$, ganz zu schweigen vom Papst selbst ${ }^{50}$. Einen letzten Angriff gegen den höfischen Brauch der étrennes hat es zu Zeiten der Französischen Revolution gegeben, nachdem die luxuriöse Dimension der Neujahrsgeschenke unter Ludwig XIV. wohl einen beträchtlichen

48 Bereits für das Konzil von Auxerre stellt Odette PONTAL mit Blick auf die Bestimmungen über Frauen, Probleme des Aberglaubens, liturgische Fragen und das Fasten mit Bestimmtheit fest, daß diese Synoden keine Spuren hinterlassen haben, siehe PONTAL 1986, S. 168, und ebd., S. 267 explizit mit Blick auf das Neujahrsbrauchtum: »Ebenso lebte die Feier des 1. Januar weiter [...] Sie wurde zwar [...] verurteilt, aber nie ausgerottet « (der an dieser Stelle in Anm. 159 gegebene Hinweis auf CABROL, Fernand: Le $1^{\text {er }}$ des calendes de janvier et la messe contre les idoles. Un épisode de la lutte du christianisme contre le paganisme, in: Origines liturgiques, Paris 1906, S. 203-210, konnte nicht nachgeprüft werden). Vgl. HELMRATH 1987, S. 335f. im Anschluß an die Erwähnungen über die Bestimmungen des Basler Konzils: »Alle [Disziplinardekrete, J.H.] beziehen sich auf die Reform des Klerus, der scanonici< und sbeneficiatic, also offenbar in erster Linie des Stiftsklerus. Die Welt der Laien wird allenfalls am Rande durch das Schauspielverbot berührt.«

49 Wie Adhémar Aleman (Kardinal und Erzbischof von Pisa), Arnaud de Corbie (Bischof von Bayeux 1375-1408 und von Evreux 1397), Gérard d'Athies (Erzbischof von Besançon 1391-1404), Gérard de Montaigu (Bischof von Poitiers 1403-1409 und 1409-1420 von Paris), Gérard du Puy (Bischof von Saint-Flour 1405-1414), Guillaume de Boisratier (Erzbischof von Bourges 1409-1421), Guillaume de Champeaux (Bischof von Laon 1419), Jean Canard, der burgundische Kanzler, dann der Cluniazenser Jean de La Grange, schließlich auch Jean Marchand (Bischof von Bethlehem und confesseur Johanns Ohnefurcht), Jean de Montagu (Bischof von Chartres von 1390-1406; Erzbischof von Sens von 1406-1415), Michel le Beuf (Bœuf) (Bischof von Lodève 1413-1430), Philippe de Moulins (Bischof von Evreux 1383-1388; Bischof von Noyon 1388-1409), Pierre Neveu (Bischof von Lavaur, dann von Alby), Pierre II d'Orgemont (Bischof von Thérouanne 1374-1384; Bischof von Paris 1384 bis 16. Juli 1409), Pierre de Savoisy (1385 Bischof von Mans; 1395-1406 Bischof von Beauvais), Raoul Cassinel (Bischof von Lodève; Bischof von Auxerre 1382-1390; Erzbischof von Reims 1390) oder Simon de Cramaud (Anhänger Clemens' VII.; Bischof von Agen 1382, Béziers 1383 und Poitiers 1385-1391 und wieder 1413-1424; als Patriarch von Alexandria 1391 erster Prälat Frankreichs; administrateur des Bistums Avignon, anschließend Carcassonnes über 18 Jahre hindurch; Erzbischof von Reims 1409; Kardinal 1413; Teilnehmer am Konzil von Konstanz).

50 Wie Amadeus VIII. von Savoyen (Felix V.), Benedikt XIII. oder Clemens VII. - Daß der Papst sich nicht scheute, den Neujahrstag für seine politischen Anliegen zu nutzen, berichtet in ihrem Aufsatz VAVRA, Elsbeth: "Te deum laudamus«. Kirchliche Feiern zur Zeit des Konstanzer Konzils (1414-1418), in: Das Fest, hg. von SCHULTZ, 1988, S. 127-139, hier S. 133. Zum Neujahrstag 1416 spendete der Papst in Konstanz Johanniswein, als ungewöhnlich notiert in der Chronik des Richenthal, wohl weil dieser Brauch des Ehren- und Liebestrunkes mit dem mit der Formel Bibe amorem beati Johannis gesegneten Weines eigentlich nur in Deutschland üblich war. Gleichzeitig gab er bei dieser Feier die Erklärung ab, er werde das Konzil nicht hindern, gegen Häretiker vorzugehen, was sich bekanntlich gegen Hus richtete. 
Umfang angenommen gehabt hatte ${ }^{51}$ : $»$ La Révolution eut la fantaisie de supprimer l'usage des étrennes comme entaché de réaction. ${ }^{52}$ Verhindert werden sollte, vergeblich, ein Weiterleben dieser höfischen Tradition, die sich dadurch auch als »gesunkenes Kulturgut « 53 zu erkennen gab: »On interdit les petites baraques des boulevards qui avaient commencé à s'y établir en 1789 et qui, avec quelques périodes d'interruptions encombrent encore chaque année Paris du 15 déc. au 15 janv. Cette tentative avorta, les mœurs furent plus fort que les lois, et les étrennes continuèrent à tenir une grande place dans les préoccupations populaires. ${ }^{54}$

Der große Schnitt allerdings, der die Fäden der traditionellen Gabenmoral zerreißen ließ, wird von Helmuth Berking durchaus richtig in der Reformation und in dem Aufkommen des Kapitalismus gesehen 55 . Die höfische Ehrengabe hingegen sei Element des letzten noch traditionellen Mustern verpflichteten Gabentausches gewesen. Ein Befund, der dann auch für die Nähe zu heutigen Schenkauffassungen spricht, wie diese den Neujahrsgeschenken zugesprochen werden können, die möglicherweise mit ursächlich dafür sind, daß gerade diese Form des Gabentausches überleben konnte ${ }^{56}$. Eine genaue Beobachtung der höfischen étrennes über das späte Mittelalter hinaus wäre freilich noch zu leisten.

51 Vgl. CaiX DE SaINT-AYMour, Amédée de: Stw. »Étrennes (hist.)«, in: La Grande Encyclopédie, Bd. 16, S. 694. Umfangreiche Angaben in: Grand dictionnaire universel du XIX $^{e}$ siècle, Bd. 7, 1870, S. 1074 (Nennung etlicher Personen am Hof Ludwigs XIV., die mit reichen Neujahrsgeschenken bedacht worden waren oder selbst als Schenker auftraten wie die Madame de Montespan 1679). Ein literarisches Relikt aus dieser Zeit ist der kleine Gedichtband von Claude François Ménestrier »Estreines de la cour«, eine Hommage an König und Hochadel: MÉNESTRIER 1659.

52 CAIX DE SAINT-AYMOUR, Amédée de: Stw. »Étrennes (hist.)«, in: La Grande Encyclopédie, Bd. 16, S. 694.

53 Vgl. zunächst NAUMANN 1922, der diesen Begriff geprägt hat, hier v.a. S. 5: „Volkskultur wird von der Oberschicht gemacht«; zur wissenschaftsgeschichtlichen Einordnung WEBER-KELLERMANN, BIMMER 1985, S. 77-85; zur kritischen Behandlung der Naumann'schen Thesen GREVERUS 1987, S. 163-167 und ASSION 1987, S. 157; differenzierte Betrachtung unter Beriucksichtigung auch umgekehrter kultureller Prozesse und Wechselbeziehungen bei WIEGELMANN 1977, S. 52 und v.a. BURKE 1981, S. 71-76.

54 CAIX DE SAINT-AYMOUR, Amédée de: Stw. „Étrennes (hist.)«, in: La Grande Encyclopédie, Bd. 16, S. 694. MÜLLER 1881, S. 504-507 berichtet davon, daß der Brauch, zum neuen Jahr étrennes zu vergeben, wegen seiner Nähe zu höfischen Gepflogenheiten 1793 verboten wurde, aber unter Napoleon auf Drängen der Händler wieder erlaubt wurde. Bezeichnenderweise fanden dann Teile der Bastille Verwendung als Neujahrsgeschenke.

55 Vgl. BERKING 1996, S. 210.

56 Gleichwohl scheint sich das Darbringen von Neujahrsgeschenken nicht stets und überall der gleichen Beliebtheit erfreut zu haben. Sylve de SAINT-HENRY zitiert die Inschrift eines Grabsteines, leider ohne datierenden Beleg: "Ci-gît dessous ce marbre blanc/le plus avare homme de Rennes/qui mourut tout exprès, le dernier jour de l'an/de peur de donner des étrennes«, siehe SAINT-HENRY 1866/1867, S. 212. 


\section{3. "Le festin du mois de janvier"}

Im Bereich der höfischen Kultur Frankreichs um $1400^{57}$ war der festlich gestaltete Austausch von Geschenken im Januar aus Anlaß des Jahresbeginns eine der typischen Begebenheiten - zumindest, folgt man gängigen Interpretationen, nach Ansicht des »höfischen« der Illuminatoren eines der Hauptwerke französischer Miniaturenmalerei, der »Très Riches Heures« des Herzogs Johann von Berry ${ }^{58}$, einem der Brüder Limburg ${ }^{59}$, der »hingerissen war vom Glanz des Hoflebens, von den Farben, dem Gold, der Eleganz, der grandiosen Phantasie. ${ }^{60}$

57 Grundsätzlich zu diesem Phänomen PARAVICINI 1994, siehe hier v.a. S. 9-19 zur ritterlich-höfischen Kultur, 40-42 zu den Wandlungen um 1400. Siehe auch die Hinweise unten S. 68 in Anm. 153.

58 Die grundlegende und äußerst materialreiche, aber schwierig zu nutzende chronologisch vorgehende Biogr. über den Herzog stammt von Françoise LEHOUX: LEHOUX, Jean de France, 3 Bde., 1966-1968. Den aktuellen Stand der Forschung gibt AUTRAND, Jean de Berry, 2000, siehe hier S. 481-484 zum jour de l'an am Beispiel des Hofes um den Herzog von Berry.

59 Wahrscheinlich Jan von Limburg, siehe Das Stundenbuch des Duc de Berry, hg. von CAZELlES, RATHOFER, 1988, S. 226. Dagegen differenziert ebd., S. 14, daß nach Raymond CAZELLES auch »Limburg B» der Maler sein könnte, mithin Hermann von Limburg (vgl. LONGNON, CAZELLES 1976 zur Urheberschaft in ihren »étapes d'élaboration des Très Riches Heures du duc de Berry «, hier u.a. im Überblick S. 29), siehe aber auch unten S. 47. Dem Herzog offenbar auf freundschaftliche Art verbunden, konnten die Brüder sich sogar den Scherz erlauben, den bibliophilen Mäzen zum Neujahrstag 1411 mit einem Buch, das nur ein angemaltes Stück Holz war, zu beschenken, AN KK 258, fol. 162v, ed. LEBEUF, Recueil, Bd. 2, 1738, S. 261; DOUËT D'ARCQ 1850, S. 167f., Nr. 82; GuIFFrEY, Inventaires, Bd. 1, 1894, S. 265, Nr. 994 und DELISLE, Recherches sur la librairie de Charles V, Bd. 2, 1907, S. 270, Nr. 297, siehe Anhang - Katalog/Jahreslisten, Nr. 1537. Aufgegriffen auch von HuIZINGA 1975, S. 381, dort zitiert nach DuRRIEU 1904, S. 81 (siehe HuIZINGA 1975, S. 512, Anm. 41), vgl. MeIss, French Painting in the Time of Jean de Berry: The Late Forteenth Century, Bd. 2, 1967, S. 48: "This gift was both a joke and a show of virtuosity." In allg. Hinsicht zu »Hofkünstlern« WARNKE 1985, hier v.a. S. 260ff., zu den Brüdern Limburg im Horizont des gegebenen Kontextes MEISS, French Painting in the Time of Jean de Berry: The Limbourgs and their Contemporairies, Bd. 1, 1974, und AVRIL 1975, S. 40-45, vgl. AUTRAND, Jean de Berry, 2000, S. 429-436.

60 POGNON 1987, S. 12. Das zu Lebzeiten des Herzogs noch nicht vollendete Werk ist, zu einem Wert von 500 l.t., notiert in einem Inventar von 1416, heute Paris SG ms. 841, Nr. 1164, als une layette de plusieurs cayers d'une très riches Heures, que faisoient Pol et ses frères, très richement historiez et enluminez, ed. GUIFFREY, Inventaires, Bd. 2, 1896, S. 280, Nr. 1164, vgl. ebd., Bd. 1, 1894, S. CLXV, Nr. 22 sowie DeLISLE, Recherches sur la librairie de Charles V, Bd. 2, 1907, S. 239, Nr. 101 und S. 290; zuerst anhand des Inventareintrags von DELISLE identifiziert, siehe DELISLE, Le cabinet des manuscrits de la Bibliothèque Impériale, Bd. 3, 1881, S. 179, Anm. 101, vgl. DELISLE 1884, S. 403. Die wichtigste Ed. ist: Das Stundenbuch des Duc de Berry, hg. von CAZELLES, RATHOFER, 1988, hier S. 14-17 die Bemerkungen zum Januarbild, denen hier größtenteils gefolgt wird. Frühere Ed. u.a.: Les Très Riches Heures du Duc de Berry, ed. LONGNON, CAZELlES, 1969 (mit einer Einfuihrung von Millard MEISS), hier zur Interpretation des Januarblattes "The Plates with 
Ob diese emphatische Formulierung in der populärwissenschaftlichen Ausgabe der "Très Riches Heures« von Edmond Pognon der tatsächlichen Begeisterung eines der Brüder Limburg entspricht, ist nicht bekannt. Schriftliche Zeugnisse dieser Art von einem der Hofmaler des Herzogs von Berry sind nicht überliefert. Daß aber der Austausch von Neujahrsgeschenken tatsächlich ein regelmäßig stattfindender Vorgang war, belegt die Überlieferung61.

Auf der ersten und größten der Miniaturen des Stundenbuchs ist der individuell porträtierte Fürst zu sehen 62 - abgesehen von dem hohen Kleriker

Commentaries. 2. January« (»we see here the day chosen for exchanging gifts «), und DURRIEU 1904 (zum Januarblatt S. 130-133; vgl. DURRIEU 1903 mit Ausfuhrungen über den Vorbildcharakter des Januarbildes für das Januarblatt des Breviarium Grimani, illustrativ hierzu auch HANSEN 1984, S. 56). MEISS selbst äußert sich in einem seiner Standardwerke der Kunstgeschichte zum Stundenbuch von Chantilly nur verschiedentlich, aber nicht dezidiert wie z.B. zu den »Grandes Heures«, vermittelt aber m.E. den besten Überblick über das einschlägige Kunstschaffen der Zeit: MEISS, French Painting in the Time of Jean de Berry: The Late Forteenth Century, 2 Bde., 1967 (nützlich ferner FIERENS-GEVAERT, La peinture en Belgique, 4 Bde., 1909-1912, und FIERENS-GEVAERT, Histoire de la peinture flamande, 3 Bde., 1927-1928, sowie insbes. PANOFSKY, Early Netherlandish Painting, 2 Bde., 1953, hier zu den »Très Riches Heures « Bd. 1, 1953, pass., v.a. S. 63-66, zum Januarbild S. 65f., Abb. in Bd. 2, 1953, Tafel 41, Abb. 88). MEISS behandelt die "Très Riches Heures « dann allerdings ausfuhhrlich in: MEISS, French Painting in the Time of Jean de Berry: The Limbourgs and their Contemporairies, 2 Bde., 1974, siehe hier Bd. 1, 1974, S. 143-224, zum Januarbild S. 188-190, Abb. Bd. 2, 1974, Nr. 539. Wichtig für den Vergleich neben den schon erwähnten Arbeiten Léopold DeLISLEs (v.a. DeLISLE 1884, hier S. 401-404) u.a. LEROQUAIS, Les livres d'heures manuscrits, 4 Bde., 1927-1943. - Einen Überblick über die Forschungen zu den "Très Riches Heures« des Herzogs von Berry gibt: Das Stundenbuch des Duc de Berry, hg. von CAZELLES, RATHOFER, 1988, S. 195-243. Eine neuere kunstgeschichtliche Studie gibt MÜLLER 2000. Zu Stundenbüchern im allg. WIECK 1988.

61 Siehe bspw. die auf die Regelmäßigkeit dieses Vorgangs verweisende Notiz in der dritten Rechnung des Jean Fraignot, receveur général des duché et comté de Bourgogne, von 1419 über die Vergabe von étrennes durch Margarete von Bayern, der Gattin Johanns Ohnefurcht, ACO B 1598, fol. 124, ed. Comptes généraux de l'État Bourguignon, Bd. 2,1, 1966, S. 471, Nr. 3956: A madame la duchesse de Bourgoingne, la somme de 280 frans, à elles bailliez et delivrez par son ordonnance et commandement, de laquelle somme elle a fait acheter les estraines par elle donnees le jour de l'an mil quatre cens et dix huit à madame de Guienne, madame d'Autheriche et mes damoiselles Anne et Agnes de Bourgoingne, aux dames et damoiselles et à aucunes gens de son hostel qu'elle a acoustumé d'estraigner chascun an [gesperrte Hervorhebung J.H.]. Siehe auch unten S. 52, Anm. 71 und S. 52, Anm. 72.

$62 \mathrm{Zu}$ den Portraits Johanns von Berry GUIFFreY, Inventaires, Bd. 1, 1894, S. CXVCXVIII, vgl. MEISS, French Painting in the Time of Jean de Berry: The Late Forteenth Century, Bd. 2, 1967, S. 68-94, der allein 66 Darstellungen nachweisen kann. Durch die den Trend am Vorabend der Renaissance bestimmende individualisierende Tendenz in der Gestaltung (vgl. die etwas pathetisch geratene Formulierung in: Feste und Feiern im alten Europa, hg. von BIEHN, 1962, S. 367: "Die volle Blüte des Festwesens hob recht eigentlich im 15. Jahrhundert an, als die ständische Ordnung des Mittelalters mit ihrem patriarchalischen Gehaben sich der künstlerischen Phantasie einer neuerwachten Individualität vermählte«) ist es möglich, den Herzog nicht nur anhand seiner Symbole zu identifizieren, sondern ihn auch aufgrund seiner Physiognomie, wie sie andere Darstellungen von ihm in ähnlicher Art auf- 
neben ihm als einziger sitzend und kenntlich unter anderem an den über seinem Kopf auf einem Baldachin angebrachten heraldischen Farben und Symbolen wie den Lilien und seinen Wappentieren, Schwan und Bär63 -, angetan mit einer diamantgeschmückten ${ }^{64}$ Pelzkappe und einer Kollane, gekleidet in eine pelzgefaßte und bestickte houppelande ${ }^{65}$, der Gäste erwartet beziehungsweise empfängt, die von einem seiner chambellans - Physiognomie und Körpergröße lassen allerdings nach einem Hinweis Werner Paravicinis vermuten, daß es sich um einen Hofnarren handelt - mit den Worten aproche, aproche hinzugebeten werden. Diese Gäste, so ließe sich in Verbindung mit dem Monat Januar nicht ohne Berechtigung annehmen66, obwohl

weisen, wiederzuerkennen, vgl. z.B. die Holzstatue in der Kathedrale St-Etienne zu Bourges. Das vermutlich erste autonome Portrait stellt möglicherweise König Johann den Guten 1350 dar und kann als Auftakt dieser Art künstlerischer Gestaltung gelten, siehe HILGER 1992, S. 18 f. mit Abb. 3 auf S. 15, die sich bemerkenswerter Weise und von Hans-Peter Hilger verständlich dargestellt vor dem Hintergrund des von William von Occam (ca. 1290-1349) erneuerten philosophischen Nominalismus herausbildete, der die individualisierende künstlerische Ausdrucksfähigkeit beförderte, siehe ebd., S. 17. Siehe zu diesem Themenbereich SCHERER 1993, S. 160-175 ("Das 14. Jahrhundert《), hier v.a. S. 165-171 zu Occam. Schmuckporträts im Rahmen der zeitgenössischen Emailkunst behandelt EICKELMANN 1984, S. 477ff.

63 Wappen, Symbole und Devisen des Fürsten werden kurz und prägnant behandelt bei GUIFFREY, Inventaires, Bd. 1, 1894, S. CXXVIIff., zu Bär und Schwan ebd., S. CXXVIIICXXXI, vgl. MEIss, French Painting in the Time of Jean de Berry: The Late Forteenth Century, Bd. 2, 1967, S. 95-97 mit weiteren Nachweisen. Eine mögliche Erklärung zu Bär und Schwan stammt von CoUderc 1927, S. 81: Berrys Patron St Ursin sei Hintergrund furr das Wortspiel ursine-oursine-ours und sine $=$ cygne. In der Abb. nicht zitiert ist die Wortdevise des Herzogs Le temps viendra.

64 Zu den Diamanten des Herzogs GuIfFREY, Inventaires, Bd. 1, 1894, S. XCIV, zu seinen joyaux de corps ebd., S. CIV. Daß der Herzog nur einen Diamanten an seiner Pelzkappe angebracht hat, mag Zufall sein, bietet aber die Möglichkeit, an dieser Stelle auf den Stellenwert mittelalterlicher Zahlenbedeutungen hinzuweisen, siehe hierzu MEIER, SUNTRUP, Lexikon der mittelalterlichen Zahlenbedeutungen, 1987 (eingängige Beispiele bei HuIZINGA 1975, S. 293f.).

65 Die houppelande, ein langer, gegürteter Überrock mit Schleppe, vorn, hinten und seitlich hoch geschlitzt, mit langen, weiten Ärmeln, war bis etwa 1450 das Staatskleid in Burgund, Frankreich und den Niederlanden, benannt nach dem südschottischen Bergland, den "uplands« (zum ersten Mal bei englischen Truppen im Elsaß ca. 1365 beobachtet). Die houppelande, extra gefertigt zum ersten Januar, unterstreicht wie auch die Anfertigung anderer Kleider den Festcharakter dieses Tages: Item pour la fourreure d'une robe entiere destarlate vermeille de IIII garnemens pour le dit seigneur [für den König?] pour la jour de la feste de novl. an, BNF ms.fr. 6743, fol. 5r. AUTRAND, Jean de Berry, 2000, S. 482 nennt die Anfertigung von 850 Orangenblättern als Schmuck für die houppelande des Herzogs von Berry just für den 1. Januar 1415. Siehe auch oben S. 43, Anm. 30 und unten S. 175, Anm. 268 und 271.

66 CAZELLES und RATHOFER vermitteln als gesicherte Erkenntnis, daß es sich um einen Neujahrsempfang handelt, siehe Das Stundenbuch des Duc de Berry, hg. von CAZELLES, RATHOFER, 1988, S. 14 (und zuletzt wieder explizit STRATFORD 1995 in ihrem Beitrag über "Das Goldene Rößl und die Sammlungen des französischen Königshofs«, hier S. 38, 41, 


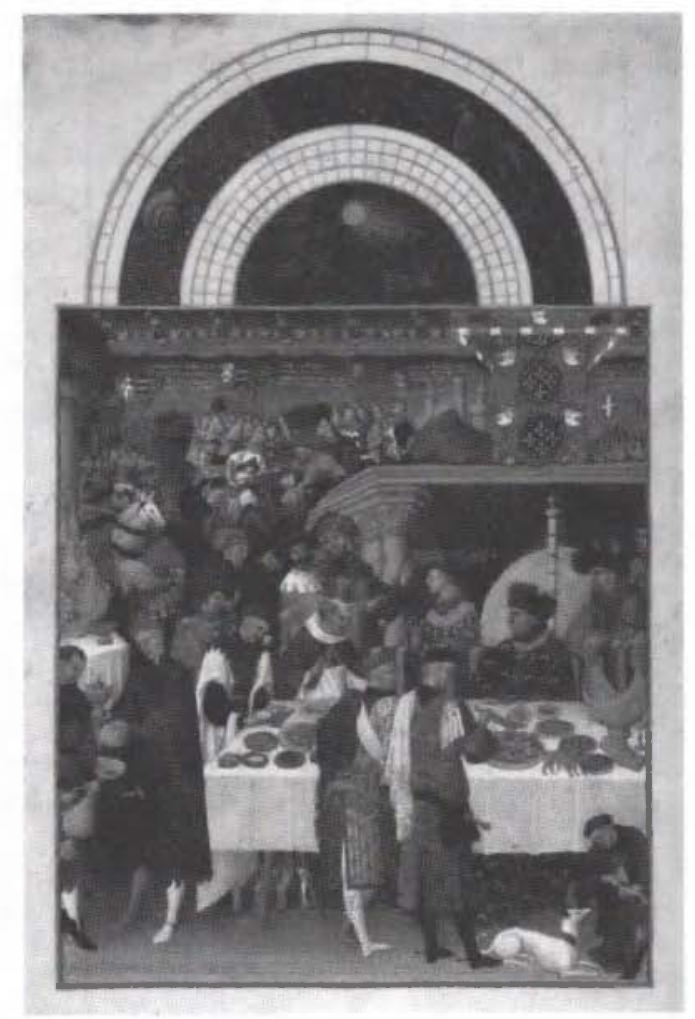

Abb. 1: Le festin du mois de janvier. Das Januarblatt in den "Tres Riches Heures« des Herzogs Johann von Berry Musee Conde, Chantilly, ms. 65, fol. $1 \mathrm{v}$ Cliche Musee Conde, Chantilly

vorsichtiger hingegen MEIss, French Painting in the Time of Jean de Berry: The Limbourgs and their Contemporairies, Bd. 1, 1974, S. 188ff.), erwähnen abèr, daß es sich nach den Forschungsergebnissen Saint-Jean Bourdins (BOURDIN, Saint-Jean: Analyses des Tres Riches Heures du duc de Berry - Identifications des personnages figurant dans le calendrier, 1, 1982, S. 141, zitiert nach: Das Stundenbuch des Duc de Berry, hg. von CazElles, RATHOFER, 1988, S. 244) auch um die Schlußsitzung der Geheimverhandlungen des Herzogs mit einer englischen Gesandtschaft vom 6. Januar 1414 handeln könnte mit dem Hinweis auf die Unwahrscheinlichkeit dieser These, würden doch die hochrangigen Personen nicht vor dem Herzog stehen, siehe auch unten S. 52-53 mit Anm. 73. COLENBRANDER 1996, S. 111 meint gar, es handele sich schlicht um eine Vorstellung des Monats Januar, in welchem man eben um das Feuer sitze; wozu sei dann festzustellen, wie AUTRAND, Le Jour de l'An, 1999, S. 6 zu Recht fragt, daß eine der abgebildeten Personen die weiße Schärpe der Armagnacs trage, zudem, siehe ebd., S. 7, der orleanistische Knotenstock zu identifizieren sei? Symbolisiert wird mithin nicht der Monat Januar, sondern die als politisches Manifest zu interpretierende Geschenkvergabe am 1. Januar, vgl. AUTRAND, Jean de Berry, 2000, S. $428-436$. 
dies in der Darstellung selbstverständlich nicht sichtbar ist, überbringen dem Herzog Glückwünsche ${ }^{67}$ und Geschenke ${ }^{68}$ und werden wohl im Gegenzug von dem Herzog selbst beschenkt worden sein. Daß der Neujahrsgeschenkverkehr tatsächlich am ersten Januar stattfand, liegt an seinen historischen Wurzeln, denn das offizielle neue Jahr, das Kirchenjahr, begann im mittelalterlichen Frankreich bis zur Anordnung Karls IX. aus dem Jahre 156469, das Jahr mit dem ersten Januar beginnen zu lassen, eigentlich mit dem Osterfest ${ }^{70}$, was in

Die Praxis der Darbringung von Glückwünschen und Dedikationen zum neuen Jahr ist für den betreffenden Zeitraum literarisch belegt, vgl. Anhang - Katalog/Jahreslisten, Nr. 867 und ebd./Nicht datierbare Angaben die Balladen, Rondeaux und Virelais von Christine de Pisan und Eustache Deschamps mit weiteren Nachweisen, siehe dazu oben S. 51ff., vgl. DAVIS 2000, S. 233f. mit weiteren Nachweisen ebd., Anm. 4. Vgl. zur volkskulturellen Seite dieses Brauchtums: Neujahrswünsche des 15. Jahrhunderts, hg. von HEITZ, 1899, SAUTER 1969 über »Neujahrswunschblätter aus sechs Jahrhunderten« (insbes. zu den Vorläufern der Neujahrswunschkarten, die, wenn es sich um Darstellungen des Jesuskindes als Einzelfigur handelt, zuerst in der nordischen Graphik des späten 15. Jh.s begegnen) und HOLTORF 1973 zu »Neujahrswünschen im Liebesliede des ausgehenden Mittelalters«, dessen Werk sich auch als »ein Beitrag zur Geschichte des mittelalterlichen Neujahrsbrauchtums in Deutschland « versteht (hier zu Neujahrsgeschenken S. 155ff.).

68 Vgl. allg. LIGHTBOWN, French Silver, 1978, und LIGHTBOWN, Secular Goldsmith's Work, 1978, S. 40f. zum Januarblatt der "Très Riches Heures«, der sich bei seinen Ausführungen zu einem nicht geringen $\mathrm{Maß}$ an den überlieferten Neujahrsgeschenken orientiert und fast alle Gegenstände aus der zeitgenössischen Produktion von Goldschmiedearbeiten behandelt, die nicht dem großen Komplex »Schmuck« zuzuordnen sind: barrils, drageoirs, écuelles, flacons, gobelets, hanaps, nefs, plats, pots, quartes, salières, tasses, tranchoirs etc. 69 Siehe GIRY 1894, S. 106. Vgl. SCHMITT, Heidenspaß und Höllenangst, 1993, S. 79 bzw. S. 79ff. (siehe auch LABORDE, Glossaire, 1872: S. 307f.). Die Gregorianische Kalenderreform erfolgte erst am 7. (15.) Oktober 1582. Poole 1934, S. 27 gibt zwei Listen mit Ländern, die den Jahresbeginn schon vor diesem Datum offiziell auf den ersten Januar legten wie Frankreich, und solchen, die sich teilweise erst sehr spät umstellten wie Venedig, das bis 1797 das Jahr mit dem ersten März beginnen ließ.

70 Genannt »Osterstil« bzw. »mos Gallicus.« Das hat auch zur Folge, daß alle jeweils zum ersten Januar eines Jahres bis Ostern gegebenen Datumsangaben die Jahresangabe des aus heutiger Sicht alten Jahres notieren (= alter Stil). Datierungen werden in der vorliegenden Arbeit durchgängig nach dem neuen, heutiger Zeitrechnung entspr. Stil gegeben, vgl. GROTEFEND, Taschenbuch der Zeitrechnung, Bd. 1, 1891: hier Stw. »Circumcisionsstil«, S. 2224, Stw. »Neuer Stik, S. 132-134 und Stw. »Neujahrstag, neujahrsabend, nienjarstag, nijarsavend «, S. 134f. Umfangreich die einschlägige Anm. S. 21-31 in: L'Art de vérifier les dates, Bd. 1, 1818, umfassend GIRY 1894, S. 103-129, v.a. S. 105f. zum ersten Januar, S. 110f. zum Osterstil Frankreichs, 112-123 zu den dort regional unterschiedlichen Stilen, knapp BOÜARD 1929, S. 302ff., hier zum Osterstil S. 304f., zum ersten Januar S. 305. Vgl. PoOLE 1934 (»The Beginning of the Year in the Middle Ages«), S. 1-27, hier S. 3-7 zur Jahresberechnung nach dem ersten Januar, 20-25 zum Osterstil, 26f. zur Wiederdurchsetzung des Januarstiles. Eine ähnliche Situation herrschte in England, wo ebenfalls zum ersten Januar Geschenke ausgetauscht worden sind, obwohl das Jahr bis 1752 am 25. März begann, siehe MYERS 1959/1960, S. 114, Anm. 114. Der historische Referenzpunkt für den ersten Januar als Jahresbeginn ist das Jahr 153 v.C., diskutiert bei MICHELS 1967, S. 97ff. mit weiteren Nachweisen. - Daneben sorgten auch andere Schenkdaten wie die Vergabe von houppelandes am 1. Mai, der daraufhin als Zeitpunkt für die Vergabe von 
der Forschung zu einer ganzen Reihe von Unsicherheiten und Unstimmigäkeiten in Datierungsfragen führte ${ }^{71}$. Auffälligerweise zeigt nun aber eben auch das Stundenbuch des Herzogs von Berry als erstes Monatsblatt den Januar und die Vergabe von Neujahrsgeschenken zum ersten Tag dieses Monats ist durch die Quellen belegt ${ }^{72}$.

Die abgebildeten Personen lassen sich, den Ausführungen Jean Longnons und Raymond Cazelles' folgend, teilweise identifizieren, zumindest aber hinsichtlich ihrer erkennbar ausgeübten Tätigkeiten, ihrer Kleidung, ihrer Amtsinsignien näher in ihren Funktionen bestimmen ${ }^{73}$. Der terminus ad quem

Neujahrsgeschenken angenommen wurde, für Verwirrung, siehe etwa PANNIER 1873, S. 218, Anm. 2: "Sur cet usage de donner des étrennes le $1^{\text {er }}$ mai $[. .$.$] voyez Jal, Dictionnaire$ critique, au mot MAI [...] « (dieses Lexikon war nicht zugänglich, so daß dieser Hinweis nicht überprüft werden konnte). GRANDEAU 1967, S. 827 berichtet in seinem Aufsatz über die Königskinder, wie diese aus Anlaß jedes größeren Festes - Ostern, Pfingsten, Allerheiligen, Weihnachten und am 1. Mai - neu eingekleidet wurden selon la coutume. Tatsache bleibt, daß der jour de l'an stets mit dem ersten Januar zu identifizieren ist, auch wenn der offizielle und kirchliche Jahresanfang ein anderer war, vgl. in dieser Hinsicht auch POOLE 1934, S. 5.

71 Siehe bspw. auch die Angaben unten S. 63, Anm. 134 zur Datierung des Schenkdatums des »Goldenen Rößls«. Vgl. die umfangreichen und im Schluß korrekten Überlegungen von Denis-François SECOUSSE in einer Anmerkung in seiner Publikation der »Ordonnances des Roys de France de la troisième race« (»Lorsqu'en France l'Année commençoit à Pasques, donnoit-on les Estrennes le jour de Pasques, ou le premier jour de Janvier? «, bezogen auf das Jahr 1362; - vgl. zur gleichen Frage POLLUCHE 1735) unter Bezugnahme auf die Stw. "Annus", "Estrennes« und "Strena« im "Glossarium mediae et infimae Latinitatis" des Charles Du Fresne Sieur DuCange, siehe oben S. 37, Anm. 3: Ordonnances des Roys de France, ed. SECOUSSE, Bd. 3, 1732, S. 583f., Anm. e. Vgl. ROCHEFORT 1735, hier v.a. S. 32ff., der in seinem "Lettre au sujet des étrennes" in Reaktion auf die Ed. der Ordonnanzen durch Secousse dessen Angaben zu den étrennes um zwei Beispiele aus der Chronik des Louis de Bourbon erweitert sehen will (siehe die chronikalischen Angaben zur Gründung des Ordens vom goldenen Schild am 1. Januar 1367: Chronique du Bon Duc Loys de Bourbon, ed. CHAZAUD, 1876, chap. II, S. 6-8, chap. III, S. 8-12, chap. V, S. 15-18, vgl. hierzu MATTEONI 1991, siehe auch unten S. 94-95).

72 Bspw. Christine de Pisan, Le Livre des Fais et Bonnes Meurs du Sage Roy Charles V, ed. SOLENTE, Bd. 1, 1936, Kap. I (Cy dit quelle fut la cause et par quel commandement ce livre fut fait): La verité est que c'est, en ce présent an de grâce mil quatre cent trois, après un mien nouveau volume, appelé De la Mutation de la Fortune, présenté, de ma part, audit très solennel prince monseigneur de Bourgogne, comme bonne étrenne, le premier jour de janvier que nous appelons le jour de l'an, lequel volume sa débonnaire douceur reçut très aimablement. Bspw. AN KK 258, fol. 112v, ed. GUIFFREY, Inventaires, Bd. 1, 1894, S. 199, Nr. 755 (vgl. PANNIER 1873, S. 389, Anm. 1): Item, une pincte de bericle garnie de II marcs II onces d'or ou environ, et le fretelet garnie de VI perles et I balay, laquelle monseigneur achata de Françoys de Nerly, marchant demorant à Paris, avec les parties des estraines que ledit Françoys delivra à mondit seigneur pour le premier jour de janvier l'an mil CCCC et IIII, la somme de IIf L francs [gesperrte Hervorhebungen J.H.]. Siehe auch oben S. 48, Anm. 61 und die vorangehende Anm. 71.

73 Nach: Les Très Riches Heures du Duc de Berry, ed. LONGNON, CAZELLES, 1969: »The Plates with Commentaries. 2. January." - Dagegen Saint-Jean BouRDIN, der aufgrund seiner These, es handele sich nicht um einen Neujahrsempfang (siehe oben S. 51, Anm. 66), folgende Personen meint identifizieren zu können: links des Herzogs sei Simon Aligret, sein 
ist dabei das Jahr 1416, das Todesjahr Johanns von Berry, dem kurz darauf Paul von Limburg im Zuge einer Pestepedemie in den Tod folgte ${ }^{74}$. Der neben dem Herzog auf der Bank sitzende Geistliche mag vor diesem zeitlichen Hintergrund der Bischof von Chartres, Martin Gouge, gewesen sein ${ }^{75}$, der im Begriff sein könnte, für erwiesene Gunst oder Gaben zu danken. Martin Gouge de Charpaignes, trésorier général nach dem Tod seines Bruders 1402 bis 1407, général des finances 1404, 1405 und 1407, dann 1408 conseiller général sur les faits des aides, war Kanzler und conseiller des Herzogs, mithin also zum fraglichen Zeitpunkt ein enger Vertrauter Johanns von Berry, der von ihm in den Jahren von 1404 bis 1416 mehrfach beschenkt worden ist ${ }^{76}$. Hinter dem Herzog stehen zwei nicht näher bestimmbare junge Prinzen oder hohe Adlige. Bei den von dem chambellan (der, wenn es sich um einen solchen handeln sollte, an Amtskette und -stab, chaîne und bâton d'office, erkennbar wäre) hinzugebetenen Personen könnte es sich zuerst um Paul von Limburg selbst handeln ${ }^{77}$, der sich somit auch selbst porträtiert haben könnte, wenn er der »höfische « der Brüder war ${ }^{78}$, mitsamt seiner Frau, Gillette le Mercier. Ein anderer der Brüder Limburg wäre in demjenigen wiederzuerkennen, der gerade im Begriff ist, seinen Durst zu stillen, ob dann allerdings Jan oder Hermann, das mag dahingestellt bleiben. Des weiteren zeigt das Bild, von links nach rechts aus Sicht des Betrachters, einen valet du gobelet und einen échanson ${ }^{79}$, am Tisch einen panetier und zwei écuyers tranchant sowie im Vordergrund einen valet $d u$ chien $^{80}$, nicht $\mathrm{zu}$ vergessen die kleinen

Arzt zu erkennen, sitzend neben ihm Guillaume Boisratier, der Erzbischof von Bourges; ferner seien anwesend die Grafen von Eu und Armagnac, die Herzöge von Bourbon-Vendôme und Savoyen und die Brüder Limburg.

74 Zur Entstehungszeit: Das Stundenbuch des Duc de Berry, hg. von Cazelles, RATHOFER, 1988, S. 225f. Siehe auch unten S. 53, Anm. 77.

75 So auch MEISS, French Painting in the Time of Jean de Berry: The Limbourgs and their Contemporairies, Bd. 1, 1974, S. 189.

76 Siehe Anhang - Katalog/Jahreslisten, Nr. 1295, 1446, 1529, 1604, 1648, 1679, 1680 und 1691 (siehe auch Nr. 1443). Vgl. GUIFFREY, Inventaires, Bd. 1, 1894, S. XLVII. Siehe unten S. 107 mit den entspr. Anm.

77 Vorsichtig in dieser Frage MEISS, French Painting in the Time of Jean de Berry: The Limbourgs and their Contemporairies, Bd. 1, 1974, S. 190, der gleichwohl S. 188 ganz entschieden für Paul als den Maler des Januarblattes eintritt. $\mathrm{Zu}$ Paul siehe AEsCHLIMANN, Dictionnaire des miniaturistes, 1940, S. 152f., und WINKLER, Friedrich: Art. "Limburg, Paul (Pol) von«, in: THIEME, BECKER, Allgemeines Lexikon der bildenden Künstler, Bd. 23, 1992, S. 227-229. - Falls es zutrifft, daß Paul von Limburg und Martin Gouge abgebildet sind, so wäre dies auch ein Indiz für die Datierung: 1411 und 1415 erscheinen beide zugleich als den Herzog von Berry Beschenkende, siehe Anhang - Katalog/Jahreslisten, Nr. 1538 und 1540 sowie $1677-1679$.

78 Siehe oben S. 47, Anm. 59.

79 Thomas de Rançon?, vgl. GUIFFrEY, Inventaires, Bd. 1, 1894, S. LXV.

80 Literatur in Auswahl, die für den Personenbestand der Zeit relevant ist, wird unten in der bibl. Vorbemerkung zum Personenindex genannt. 
Tischhunde und den weißen Windhund ${ }^{81}$. Die französische Historikerin Françoise Autrand datiert die Szenerie mit Blick auf die sich im Januarblatt widerspiegelnden politischen Konstellationen der Zeit unter Verwendung der Inventare des Herzogs auf $1415^{82}$ und siedelt sie in der von ihr so bezeichneten Phase der »réconciliation ${ }^{83}$ an, nachdem sich Bourguignons und Armagnacs am 4. September 1414 im Vertrag von Arras einmal mehr zur Einhaltung des Friedens von Chartres von 1409 verpflichtet hatten. Ist dies tatsächlich der Fall, dann finden sich die Personen, die abgebildet sind, auch unter denjenigen, die den Herzog an diesem Tag reich beschenkten, und sind in seinem Inventar notiert ${ }^{84}$. Berry erhielt 1415 von Adhémar Aleman, dem Kardinal und Erzbischof von Pisa ${ }^{85}$, Arnoul Belin, dem trésorier der SainteChapelle von Bourges, Bonne d'Artois und Charles d'Artois, seinen Enkeln, von Constantin de Nicolas, einem italienischen Händler, und seinen Sekretären Erard Moriset, Michel le Beuf, Oudart de La Barre und Pierre de Gynes, von Guillaume de Boisratier, dem Erzbischof von Bourges, von Guillaume de Champeaux, seinem maître des requêtes de l'hôtel, von Guillaume de Lodes, einem seiner chambellans, und Guy de La Roche-Guyon, einem chambellan des Dauphins, von Jacques II de Bourbon, von Jean de La Barre, einem général conseiller des finances, von seiner Frau, der Herzogin Jeanne de Boulogne, von Johann I. von Bourbon, Karl von Orléans und Louis de Vendôme, von Ludwig dem Bärtigen und dem Dauphin Ludwig von Guyenne, von seiner Tochter Marie de Berry, von Martin Gouge und Paul von Limburg, von Pierre Culon, einem seiner maîtres der Chambre aux deniers, von Pierre de l'Esclat, einem der hohen Räte der Monarchie, der 1418 während der burgundischen Besetzung von Paris ums Leben kommen sollte,

81 Hierzu DuRRIEU 1909. Über die ménagerie Berrys hat Jules-Joseph Guiffrey gearbeitet: GUIFFREY 1899, vgl. GUIFFREY., Inventaires, Bd. 1, 1894, S. CXXV-CXXVII. Nach AN KK 258, fol. 67v, ed. GUIFFREY 1899, S. 77 gab der Herzog 1375 a un valet des chiens de la Royne, qui a présenté à Mgr. un lévrier blanc de par la Royne 6 l.t., vgl. GUIFFREY 1899, S. 68. Einen weiteren weißen Windhund erhielt Johann von Berry 1400 von der Herzogin von Bourbon, siehe Anhang - Katalog/Jahreslisten, Nr. 1018.

82 AUtRand, Le Jour de l'An, 1999, S. 6, siehe auch oben S. 51, Anm. 66. - Françoise AUTRAND stellte freundlicherweise ihren für das "Bulletin de la Société Nationale des Antiquaires de France« im Druck befindlichen Aufsatz »Le Jour de l'An 1415 à la cour du duc de Berry« im Manuskript zur Verfügung. Siehe zur Datierung auch oben S. 53, Anm. 77. 83 AUTRAND, Le Jour de l'An, 1999, S. 6.

84 Vgl. AUTRAND, Jean de Berry, 2000, S. 452-455.

85 Dessen Geschenk mag von Paul von Limburg überbracht worden sein, siehe AUTRAND, Le Jour de l'An, 1999, S. 10. Adhémar Aleman war als Legat des Heiligen Stuhls 1410 von Johannes XXIII. nach Frankreich entsendet worden und hatte große Anstrengungen unternommen, den Frieden zwischen Armagnacs und Bourguignons herzustellen. 
von Pierre Neveu, dem Bischof von Alby, von seinem Buchbewahrer Robinet d'Estampes und seinem Arzt Simon Aligret insgesamt 27 Geschenke ${ }^{86}$.

Die Szene spielt mit an Sicherheit grenzender Wahrscheinlichkeit in einer der Residenzen des Herzogs, möglicherweise in Poitiers, vergleicht man den Kamin mit dem noch heute dort erhaltenen ${ }^{87}$, vor dessen Hitze sich der Fürst mit einem Schirm aus Korbgeflecht geschützt haben könnte ${ }^{88}$, und hat den Charakter eines Festmahls ${ }^{89}$. Theodor Straub, der Biograph Ludwigs des

86 Siehe Anhang - Katalog/Jahreslisten, Nr. 1655-1667, 1669, 1672, 1674-1676, 16781681, 1683-1687. Es war dies der Neujahrstag, an dem der Herzog überhaupt am reichsten beschenkt worden war, vgl. AUTRAND, Jean de Berry, 2000, S. 428.

87 Siehe die Abb. bei Favreau 1991, S. 122, Nr. 3, und die Photographie bei Hilger 1992, S. 21, Abb. 8. Dies ist allerdings nur eine Vermutung, denn eine Betrachtung der Aufenthaltsorte des Herzogs im fraglichen Zeitraum zeigt, daß sich der Herzog hauptsächlich in Paris aufhielt, dort war das Hôtel de Nesle sein Stadtpalais, vgl. zum Itinerar LEHOUX, Jean de France, Bd. 3, 1968, S. 423-513. Der »königliche« Palast in Bourges war die eigentliche Herzogsresidenz, daneben hatte Johann von Berry noch eine ganze Reihe weiterer Residenzen, darunter die Schlösser Bicêtre (vgl. BEAUCHAMP 1904-1906) oder Mehun-sur-Yèvre (MEISS, French Painting in the Time of Jean de Berry: The Late Forteenth Century, Bd. 2, 1967, S. 291 spricht nicht ohne Berechtigung vom "wealthy nomad, wandering from one château to another«). Saint-Jean BOURDIN (siehe oben S. 51, Anm. 66) vermutet als Ort der Abb. das Hôtel de Giac, siehe Das Stundenbuch des Duc de Berry, hg. von CAZELLES, RATHOFER, 1988, S. 14.

88 Dieser Umstand verweist auf die Jahreszeit, die nahezu zwangsläufig größere Feste im Freien unmöglich gemacht hat. Häufig wurde der Winter, zumindest die Zeit von etwa Weihnachten bis Neujahr, fast ortsfest in einer der Residenzen verbracht, bestätigt durch die Itinerare, siehe Anhang - Neujahrstage und Aufenthaltsorte 1381-1422. Vgl. Pierre ALEXANDREs quellennahe Zusammenstellung über das europäische Klima von 1000 bis 1425: ALEXANDRE 1987, insbes. S. 520-575 (siehe neuerdings GLASER 2001, hier v.a. der allerdings auf den deutschen Raum konzentrierte Abschnitt S. 61-92, in welchem S. 67f. die Zeit des Übergangs vom 14. zum 15. Jh. behandelt wird). Daraus resultiert dann möglicherweise auch die Tatsache, daß Neujahrsgeschenke in einer großen Zahl von Boten überbracht wurden, siehe Anhang - Katalog/Jahreslisten, pass., so daß an den Wendungen envoyé par oder donné par in einer Vielzahl der Fälle abgelesen werden kann, wer mit wem bei dem Schenkakt am Neujahrstag zusammen war und wer nicht. Siehe auch unten Kap. C. II. 1. b.

89 Entspr. dem Titel, den Paul DURRIEU der Abb. gegeben hat: Le festin du mois de janvier, DURRIEU 1904, S. 130, vgl. HEINISCH 1994, S. 115 im Kontext von »Fast and Feast.« Die gleichwohl spärliche Überlieferung zum Festverlauf scheint dies nicht zufällig zu bestätigen. Der »Mesnagier de Paris« erwähnt am Beispiel der Feste am Hof des Herzogs von Berry die großen Schlemmereien, vgl. AUTRAND, Jean de Berry, 2000, S. 481. Festmahl und Turnier fanden 1386 in Toulouse am 1. Januar statt, LEHOUX, Jean de France, Bd. 2, 1967, S. 155, Anm. 3. Philipp der Kühne wiederum war bspw. am 1. Januar 1383 souper et gister à Noyon devers le roy, BNF, Coll. de Bourgogne, vol. LIII, fol. 9r; am 1. Januar 1397 disna à l'ostel monseigneur de Berry, ACO B 326; am 1. Januar 1399 disner à Paris, en l'ostel l'evesque, ACO B $1511^{\text {bis }}$ (ohne fol.-Angabe zitiert nach PETIT 1888, S. 282); am 1. Januar 1401 disner à Corbueil, ACO B $1522^{\text {bis }}$, fol. 40r. Vgl. für Burgund bei COMMEAUX 1979 den Abschn. über die Etikette bei Tisch, S. 227-229, und ebd., S. 229-236 über »Les banquets«. In diesen Zusammenhang gehört auch die Bedeutung der Gabe im Rahmen der Gastfreundschaft, dazu insbes. BERKING 1996, S. 137-184 in seiner "Anthropologie des Gebens«, vgl. BETH, Karl: Art. "Gastfreundschaft«, in: HdA, Bd. 3, 1930/1931, S. 307-312, 
Bärtigen, des Bruders der Königin Isabeau, verlegt eine derartige Feier, wie sie Anfang des Jahres 1412 am burgundischen Hof stattgefunden haben könnte, nicht nur auf die Tage vom 3. bis zum 5. Januar, »zu denen der Dauphin Ludwig, die Dauphine Margarete, die Schwiegertochter Michelle von Frankreich, der Bruder Philipp von Nevers, Graf Peter von Mortain und zahlreiche große Herren und Damen - mit Ausnahme des Königs und der Königin erschienen «, sondern erweitert sie auch um die Dimension, daß mnach Tisch bis tief in die Nacht hinein getanzt und gefeiert wurde. ( $^{90}$ Zahlreiche Belege bestätigen die Anwesenheit von Musikern ${ }^{91}$. Es schien aber nicht die Regel gewesen zu sein und auch nicht zwingende Notwendigkeit, daß die Teilnehmer des Geschenkverkehrs persönlich zusammentrafen. Ersichtlich ist dies an den Itineraren ${ }^{92}$ und an den häufigen Botenentlohnungen ${ }^{93}$. Daß ein Fürst Geschenke selbst übergeben haben sollte, ist zudem anzuzweifeln ${ }^{94}$.

Einen Teil der Gegenstände, die, wie auszuführen sein wird, auch als Geschenke Verwendung finden konnten, können auf dem Bild erkannt werden. So ist - wiederum aus Sicht des Betrachters - auf einem Tisch an der linken Seite des Bildes, in den Händen der beiden links im Vordergrund

HILTBRUNNER, Otto: Art. »Gastfreundschaft«, in: RAC, Bd. 8, 1972, Sp. 1061-1123 und SIEVERS, Kai-Detlev: Art. "Gastfreundschaft«, in: HRG, Bd. 1, 1971, Sp. 1389-1391, dann HELLMUTH 1984, auch: Gastfreundschaft, hg. von PEYER, MüLLER-LUCKNER, 1983, sowie PEYER 1987, grundsätzlich ALTHOFF 1990, S. 203-211.

90 Vgl. STraub 1965, S. 123 mit Anm. 499 in Anlehnung an PETIT 1888, S. 385f., siehe Anhang - Katalog/Jahreslisten, Nr. 1553. Vgl. auch STRAUB 1965, S. 131 mit Anm. 516 in Anlehnung an PETIT 1888, S. 396. Zur Tageszeit (besser: Nachtzeit) COREMANS 1844, S. 37 mit Blick auf das »belgisch«-germanische Altertum (COREMANS' Anliegen war eine Ergänzung der Arbeiten der Gebrüder GRIMM): "Toutes des fêtes de la nuit-mère et des douze nuits [...] se célébraient pendant la nuit: c'étaient des banquets, richement éclairés, ou en ne mangeait que certains mets [...].«

91 Die natürlich auch von diesem Fest profitierten, siehe Anhang - Katalog/Jahreslisten, bspw. Nr. 182-184, 236, 301, 892, 895, 903 oder 1384. Die Geschenke, die die Musiker erhielten, waren ein nicht unwesentlicher Bestandteil ihrer Einnahmen, vgl. MARIX 1974, S. 90: "A ces gages s'ajoutent une pension [...] et la moitié des deniers donnés en largesse (l'autre moitié étant réservée aux officiers d'armes, sroys, héraults et poursuivants ), à l'occasion des fêtes de Noël, du Jour de l'an, de Pâques, des réceptions et des voyages.« Zur Musikgeschichte der Zeit und v.a. den Musikern des Herzogs Berry GÜNTHER 1963, dann PIRRO 1930, MARIX 1939 (mit Blick auf Burgund) und in allg. Hinsicht ZAK 1979, leider nur den Zeitraum von etwa 1150 bis cirka 1300 erfassend. Eine umfangreiche Bibl. bietet KISBY 1998. Vgl. RIEGER 1987, S. 29 mit Anm. 6 und weiteren Nachweisen zur musikalischen Begleitung des Mahles. Ergänzend heranzuziehen sind HARTUNG 1982, hier S. 72ff. zu den Einkünften der Spielleute, zu denen eben auch Geschenke zählten, und DOBOZY 1992.

92 Siehe Anhang - Neujahrstage und Aufenthaltsorte 1381-1422. Vgl. auch oben S. 55, Anm. 88.

93 Siehe auch unten Kap. C. II. b und die zahlreichen Angaben in Anhang - Katalog/Jahreslisten, pass.

94 Siehe aber exemplarisch die auf dem Umschlag der vorliegenden Arbeit dargestellte Überreichung von Geschenken durch Karl V. an Kaiser Karl IV. Anfang 1378. 
stehenden Bediensteten und auf der großen Tafel, die mit einer weißen Tischdecke aus Damast ${ }^{95}$ gedeckt scheint, goldenes oder vergoldetes und silbernes oder versilbertes Tafelgeschirr ${ }^{96} \mathrm{zu}$ sehen - vaisselle d'or, doré, d'argent, vermeil - wie Teller, Krüge (pots und Aquamanilen, aiguières), Pokale (hanaps oder gobelets), Platten, und Tafelschiffe (nefs), hier ein Pavillon-Salzbehälter (salière $d u$ pavillon). Der linke Tisch ${ }^{97}$ hatte im gegebenen Zusammenhang sicher auch repräsentative Funktion, war aber hier das zu jedem Festmahl gehörende Prunkbuffet, keineswegs ein "Gabentisch" für die Zurschaustellung geschenkter Gegenstände ${ }^{98}$.

In seinen Ausführungen zu den Festen des deutschen Adels am Ausgang des Mittelalters hat Andreas Ranft die zentralen Elemente eines solchen höfischen Festes listenförmig erfaßt ${ }^{99}$. Zur Form gehörten die ;festlichgastliche Tafel « ${ }^{100}$, musikalische ${ }^{101}$ und andere Unterhaltung mit Tanz, das Turnier als Höhepunkt und schließlich der Gottesdienst ${ }^{102}$ mit allen dazugehörigen Attributen ${ }^{103}$, natürlich die Festteilnehmer, und zwar nicht nur der Fürst und der Adel, sondern auch das Personal, sowie ein die Öffentlichkeit präsentierendes Publikum ${ }^{104}$. Bis auf das jahreszeitlich bedingte Fehlen eines Turniers (stellvertretend befindet sich im Hintergrund des Bildes eine Tapisserie mit der Darstellung einer Schlacht ${ }^{105}$ ) finden sich alle Elemente in der oben geschilderten Szene wieder. Die tatsächliche Anwesenheit von Publikum nach heutigem Verständnis ist für das Herstellen von Öffentlichkeit, zumal bei der durch die Quellen belegten Anwesenheit von Gesandten und Boten, tatsächlich abdingbar. Zweifelsfrei wirkte eine real existente Öffentlichkeit als »Festverstärker«106, aber unter Öffentlichkeit »ist selbst-

102 Möglicherweise hängt die Anwesenheit des Geistlichen, ob es nun Martin Gouge oder ein anderer war, auch damit zusammen, daß der erste Januar am Hof nicht nur als weltliches Fest begangen wurde, sondern daß an diesem Tag offensichtlich auch eine Messe stattfand, siehe AN KK 254, fol. 48v (in der Rubrik Menuz Dons [...] et offrandes) die Notiz von geringen Geldgeschenken für diejenigen, die an 1. Januar 1400 in Bicêtre die Messe ausgerichtet hatten, siehe ebd., fol. $103 \mathrm{v}$ denselben Vorgang für 1401 in Poitiers. Vgl. AN KK 30 (Rechnung der chambre aux deniers Karls VI.), fol. 24v für den 1. Januar 1387: [...] grant messe, a la sainte chappelle du palais, le premier jour de l'an.

103 RANFT 1995, S. 245-247.

104 Ebd., S. 248-250.

105 Siehe hingegen oben S. 55 in Anm, 89 den Hinweis auf ein Turnier, das am 1. Januar 1386 in Toulouse stattfand.

106 Nach einer von Colette BEAUNE versuchten Einteilung der höfischen Feste müßte der festin du mois de janvier zu den privaten Festen gezählt werden wie auch Bälle und Jagden 
verständlich nicht die nahezu totale Öffentlichkeit der Gegenwart gemeint, sondern die selektive, qualifizierte Öffentlichkeit der Herrschaftsträger, ohne die kein Fürst etwas ausrichten kann.«107 Wesentlich sind Ranfts kurze Erörterungen zu den Funktionen adliger Festkultur, wie sie auch für den festin du janvier gelten: Durchsetzung sozialer Exklusivität, das Nebeneinander von hierarchisierenden und egalisierenden Elementen als soziales Regulativ 108 und herrschaftlicher Lebensgestus als herrschaftslegitimierendes Instrument ${ }^{109}$.

im Unterschied zu den öffentlichen wie Weihen, feierliche Einzüge (vgl. bspw. BAPST 1891) oder Begräbnisse: BEAUNE 1989, S. 115. Allein die Anwesenheit von Angehörigen anderer Höfe dokumentiert jedoch in hinreichender Weise, daß es sich bei dem festin du mois de janvier um ein im zeitgenössischen Sinn durchaus öffentliches Fest handelte. Ein Begriff von Öffentlichkeit, der auf die Anwesenheit des "Volkes« abzielt, wird dem zeitgenössischen Begriff von Öffentlichkeit freilich nicht gerecht. HANNIG 1986, S. 154f. äußert sich zu den Trägern von Öffentlichkeit im Rahmen des frühmittelalterlichen höfischen Gabentausches eindeutig: »Dort gilt, daß alle Geschenke söffentlich sind und vor dem Kreis der Sippe, der Gefolgsleute, der auf den Reichs- und Hoftagen versammelten Großen oder dem Hofstaat vollzogen werden." Vgl. auch die Überlegungen KAMINSKY 1993. Erst vor diesem Hintergrund macht die Beziehung von Fest, Gemeinsamkeit und Öffentlichkeit Sinn, wie OECHSLIN 1992, S. 9 nach GADAMER 1977, S. 52ff. hervorhebt: "Wenn etwas mit aller Erfahrung des Festes verknüpft ist, dann ist es dies, da $B$ es jede Isolierung des einen gegenüber dem anderen verweigert. Das Fest ist Gemeinsamkeit und ist die Darstellung der Gemeinsamkeit selbst in ihrer vollendeten Form. Fest ist immer für alle.« - Siehe auch die nachfolgende Anm. und S. 140, Anm. 19. Vgl. allg. zu dem großen Forschungsbereich Feste und Feiern: Essen und Trinken in Mittelalter und Neuzeit, hg. von BITSCH, EHLERT, ERTZDORFF, 1987, hier v.a. die Beiträge ALTHOFF 1987 (vgl. ALTHOFF 1990, S. 203ff. über »Mähler und Feste (), RIEGER 1987 und ZIMMERMANN 1987, dann HEERS 1987, hier v.a. dessen einleitende Darlegungen S. 9-33, HEERS, Jacques: Art. »Feste. A. Lateinischer Westen«, in: LexMA, Bd. 4, 1989, Sp. 399-405, und Feste und Feiern im Mittelalter, hg. von ALTENBURG, JARNUT, STEINHOFF, 1991, hier v.a. den Beitrag ALTHOFF 1991.

107 PARAVICINI, Werner: Zeremoniell und Raum, in: Zeremoniell und Raum, hg. von DEMS., 1997, S. 11-36, hier S. 15. Und hier gilt, siehe ebd., daß »im Vordergrund der öffentlichen Kommunikation nicht der verbale Diskurs [steht]. Auf Repräsentation - nicht im Sinne von Stellvertretung, sondern von Zur-Schau-Stellung - zielte das Verhalten der Herrschafts- und Funktionsträger in mittelalterlicher Öffentlichkeit.« Vgl. DANIEL 2000, S. 49. Weitere grundsätzliche Überlegungen bei LAMBRECHT 2000.

108 Hinsichtlich der Tatsache, daß der Geschenkverkehr zum neuen Jahr wohl in der Regel die Form eines festlichen Banketts hatte, sei hingewiesen auf die These von Dorothea BAUDY am Beispiel der antiken Neujahrsgeschenke, daß durch diese das soziale Ranggefuige eher bestätigt (Sitzordnung!) als verändert worden sein dürfte, aber daß die soziale Verpflichtung und das ungeschriebene Gesetz der Ehre gerade vom Ranghöheren Großzügigkeit fordere, die sie gestützt sieht durch die Ergebnisse der Untersuchung von Gerhard J. BAUDY über die Tischordnung als Wurzel sozialer Organisation: BAUDY 1983, hier v.a. S. 142, zitiert nach BAUDY 1987, S. 7 mit Anm. 34. Siehe hierzu BERKING 1996, S. 116136.

109 RANFT 1995, S. 252-256. - Hinsichtlich des genannten Personenkreises ist mit Jacques HEERS einer der Hauptzwecke eines solchen Festes anzudeuten: Bindung von Klientel und sozialer Anhängerschaft, vgl. HeERS 1987, S. 22, in allg. Hinsicht PFLÜCKE 1972 zur Soziologie der Gefolgschaft. 
Im Fall des Herzogs von Berry sind es dessen Inventare ${ }^{110}$, die uns entsprechend seiner angenommenen Rolle als Empfangender auf dem Januarbild 111 - die Entfaltung dieses herrschaftlichen Lebensgestus' vor Augen führen 112 , und die die Grundlage für die Beurteilung der überragenden Stellung geben, die der Herzog in der Geschichte des Mäzenatentums als offensichtlich leidenschaftlicher Sammler spielt ${ }^{113}$. Hier finden sich idealer-

110 BNF ms.fr. 11496 (2. Dezember 1401-23. Februar 1403, begonnen von Guillaume de Ruilly, beendet von Robinet d'Etampes; ed. GUIFFREY, Inventaires, Bd. 2, 1896, S. 1-166), AN KK 258 (erstellt von Robinet d'Etampes, beinhaltet unter den Nr. 1-1099 den Stand bis 31. Januar 1413 und unter den Nr. 1100-1251 die Zugänge vom 1. Februar 1413 bis zum 16. Juni 1416; ed. GUIFFREY, Inventaires, Bd. 1, 1894, S. 1-336), BNF ms.fr. 20924 (Inventaire de la librairie [1402] et de la chapelle [1416] de Jean de France, duc de Berry) und BNF n.a.fr. 1363 (État des joyaux, ornements, livres et autres objets remis à Arnoul Belin, trésorier de la Sainte-Chapelle du palais du Bourges, par Robinet Destampes, garde des joyaux du Duc de Berry, 1404-1407, entspricht der von BEAUVOIR 1855 und 1860 benutzten Kopie Bourges, Archives du Cher, liasse 8 G $1452 \mathrm{M}$ ), alle zumindest zum Teil zu benutzen in der Ed. GUIFFREY, Inventaires, 2 Bde., 1894-1896. Ebd., Bd. 1, 1894, S. LVf., Anm. 1 wird eine kleine jahrweise geführte Statistik der Geschenke gegeben, die der Herzog von Berry de hauts personnages ou de familiers erhielt unter expliziter Nennung der Neujahrsgeschenke und einer abgesetzten Auflistung der erhaltenen Hss., siehe auch unten S. 102f. mit Anm. 145. Siehe weiterhin die "Catalogues de librairies du Louvre et du duc de Berry", BNF n.a.fr. 10827, ed. DELISLE, Le cabinet des manuscrits de la Bibliothèque Impériale, Bd. 3, 1881, S. 114-195.

111 Aktuell mit Blick auf diese Szene und auf Grundlage v.a. der Ed. GulFrREY, Inventaires, 2 Bde., 1894-1896: AUTRAND, Le Jour de l'An, 1999.

112 Luxus als Prestige- und Repräsentationsfunktion ist unabdingbares Strukturelement aller Höfe (noch Jules LABARTE sah den höfischen Luxus am Beispiel des „Goldenen RöBls« einzig in den charakterlichen Eigenschaften Karls VI. begründet, LABARTE 1869, S. 5), auch des päpstlichen, vgl. MÜNTZ 1899 und MOLLAT, G. 1958, S. 281 mit einigen Zahlen zum finanziellen Aspekt. Eine eigene Geschichte des Luxus' stammt von Henri BAUDRILART, Histoire de luxe, 4 Bde., 1878-1881, siehe hier Bd. 3, 1980, S. 280-322. KRAEMER, Pierre: Le luxe et les lois somptuaires au moyen âge, Paris 1920, lag leider nicht vor.

113 Vgl. SCHLOSSER 1908 über die »Kunst- und Wunderkammern der Spätrenaissance«, hier v.a. S. 22-119 Kap. II. "Die Kunst- und Wunderkammern « und hier S. 22-35 zu deren mittelalterlichen Ursprüngen; S. 23-32 zum Schatz und der Rolle Johanns von Berry als Sammler. Siehe auch den kurzen Art. FILLITZ 1963. Vgl. Umberto ECOs Reflexionen über die "Sammelleidenschaft« hinsichtlich einer spezifisch mittelalterlichen »ästhetischen Sensibilität« im Spannungsfeld der Auffassungen von Kunst und Schönheit: ECO 1991, S. 27-32, hier v.a. S. 30. Später einsetzend, aber die mit den fürstlichen Schatzkammern einsetzende Entwicklung zum (modernen) Museum als »Geschichte der Kunstkammer« in spannender Form vermittelnd: BREDEKAMP 1993. Siehe auch HABSBURG 1997 über die fürstlichen Kunstkammern in Europa, hier v.a. Kap. 1 zu den Valois-Sammlungen, dort wiederum zu Johann von Berry, S. 15-19. - Zu dem großen Bereich des Mäzenatentums siehe zunächst für den allg. Überblick MOLLAT 1985 und MOLLAT, Michel: Art. »Mäzen, Mäzenatentum«, in: LexMA, Bd. 6, 1993, Sp. 430-433. In literaturhistorischer Hinsicht wichtig u.a. MOORE 1913 und HOLZKNECHT 1923 - zu den Arbeiten von Joachim BUMKE siehe unten S. 100, Anm. 141; zur »Rolle des Auftraggebers in der Kunst« HIRSCHFELD 1968. Für die Rolle der Höfe als Orte des Mäzenatentums sind heranzuziehen die verschiedenen Arbeiten in: 
weise nicht nur Punkt für Punkt detaillierte Beschreibungen der Gegenstände, die sich im Schatz des Herzogs zum Zeitpunkt der Abfassung befunden haben, sondern darüber hinaus hinsichtlich der als solche gekennzeichneten Neujahrsgeschenke auch das jeweilige Datum und der Name des Schenkers. Die Überlieferung von den den Herzog betreffenden Rechnungsbeständen ist allerdings äußerst fragmentarisch ${ }^{14}$. Selten ist der Überlieferungszufall allerdings so günstig, daß wir über die Neujahrsgeschenke nicht nur aus den einschlägigen Quellen der Zeit - überwiegend Überreste im klassischen Droysen'schen Sinn 115: Rechnungsbestände wie Zahlungsanweisungen, Quittungen, Halbjahres- und Jahresabrechnungen oder Inventare 116 - erfahren, sondern - abgesehen von einer nicht geringen Zahl an (illuminierten) Handschriften ${ }^{117}$ - diese selbst heute noch bewundern können ${ }^{118}$. Meist hatten gerade Edelmetalle ${ }^{119}$ und Edelsteine ${ }^{120}$ sowie die wertvollen Gold-

Europas Fürstenhöfe, hg. von DICKENS, 1978, zum zeitlichen Vergleich SALET 1985 und Patronage in der Renaissance, hg. von LYTLE, ORGEL, 1981.

114 AN KK 250 (Compte de la trésorerie du duc de Berry tenu par Macé Heron, trésorier général du Duc, 1413-1414), ed. TOULGOËT-TRÉANNA 1889/1890, zum Teil ed. GUIFFREY, Inventaires, Bd. 2, 1896, S. 331ff. und GUIFFREY 1899, AN KK 251 (Comptes d'Étienne Valée, maître de la chambre aux deniers, 1370-1373), ed. TOULGOËT-TRÉANNA 1889/1890, zum Teil ed. GUIFFrEY, Inventaires, Bd. 2, 1896, S. 320ff. und GUIFFREY 1899, AN KK 252 (Comptes de Nicolas Mangin, maitre de la chambre aux deniers, 1374-1378), ed. TOULGOËT-TRÉANNA 1889/1890, zum Teil ed. GUIFFrEY, Inventaires, Bd. 2, 1896 und GUIFFrEY 1899, AN KK 253 (Comptes de Philippon de Veauce et de Jean de Ruilly, successivement maîtres de la chambre aux deniers, 1397-1399), ed. TOULGOËT-TRÉANNA 1889/1890, zum Teil ed. DOUËT D'ARCQ 1865, GuIFFREY, Inventaires, Bd. 2, 1896 und GUIFFREY 1899, AN KK 254 (Chambre aux deniers, 1399-1401), Forts.: BNF ms.fr. 6744, ed. TOULGOËT-TRÉANNA 1889/1890, zum Teil ed. GUIFFREY, Inventaires, Bd. 2, 1896 und GUiFFREY 1899, AN KK 263 (Chambre aux deniers, 1395-1396), BNF ms.fr. 6747 (Comptes du duc de Berry, fol. 1: Compte de Maurice Claveurez, 1412; fol. 25: Compte de Lomer le Bez, 1418 [Verkaufsliste pour le fait de nostre guerre [...] et non pour autre cause], ed. fol. 25-97: HENWOOD 1978 und BNF ms.fr. 10369 (Fragments de comptes des $X^{2} V^{e}-X V^{e}$ siècles, hier zu Berry fol. 1: 1410, fol. 53: 1385-1386 und fol. 65). - Siehe unten Abschn. III. »Der Quellenbestand", hier zur archivalischen Überlieferung unter 1 .

115 Siehe DROYSEN 1943, S. 38-50, hier v.a. S. 47-50, vgl. BERNHEIM 1912, S. 102-110, hier v.a. S. $107 f$.

116 Siehe zum Quellenbestand im vorliegenden Kap. Abschn. III.

117 Hierzu unten S. 100ff.

118 Siehe zu diesem Problem die Äußerungen bei FRITZ 1982, S. 21-36 (»Verlorenes und Erhaltenes«). Auch die chronikalische Überlieferung ist äußerst dünn, siehe hierzu unten S. $91 \mathrm{ff}$.

119 Siehe ElBERN, Victor H.: Art. »Gold. IV. Wertschätzung, Würde, Metaphorik«, in: LexMA, Bd. 4, 1989, Sp. 1537-1538 (vgl. L'or au moyen âge, 1983) und ELBERN, Victor H.: Art. "Silber. III. Wertschätzung, Metaphorik«, in: ebd., Bd. 7, 1995, Sp. $1900 \mathrm{ff}$. (hierzu allg. LightBOWN, Ronald W.: The migration period and the later middle ages, in: BLAIR 1987 , S. 37-65, v.a. S. 57-65 zur "gothic period»).

120 Behandelt unten in Kap. C. II. 1. 
schmiede- und Goldschmiedeemailarbeiten ${ }^{121}$ und sonstige wertvolle Materialien (zum Beispiel Elfenbein ${ }^{122}$ ), aber auch Bilder ${ }^{123}$ und Tapisserien ${ }^{124}$, Stoffe ${ }^{125}$, Kleidung ${ }^{126}$ und Pelze ${ }^{127}$ oder Reliquiare und Reliquien ${ }^{128}$ sowie Exotika (zum Beispiel die im Inventar des Herzogs aufgeführten »Hörner des Einhorns«, die Stoßzähne des Narwals ${ }^{129}$, oder der »Verlobungsring Josephs ${ }^{\prime 130}$ ) eine ganz erhebliche Bedeutung als finanzielle Reserve, die häufig nach dem Tod ihres Besitzers - zu Geld gemacht wurde, eingeschmolzen oder direkt verkauft, um mögliche Engpässe zu überwinden. So bestimmte beispielsweise eine Ordonnanz Karls VI. von 1417 nach dem 1416 erfolgten Tod Johanns von Berry, eine sassez grand quantité d'orfèvrerie d'or et d'argent« zu verkaufen »pour payer les soldats qui luttaient contre les Anglais «131. Von den genannten Gegenständen selbst ist mithin fast nichts

121 Behandelt unten in Kap. C. II. 1. Zum »Goldenen Rößl« unten S. 62-64.

122 Im Rahmen des Geschenkenverkehrs zum neuen Jahr fand dieses Material allerdings nur ein Mal Verwendung, siehe Anhang - Katalog/Jahreslisten, Nr. 1408: une grant fleur de lis d'argent doré, qui se ferme à charnières, en laquelle a par dedens la Vie et Passion Nostre Seigneur et plusieurs sains, tout fait d'ymaiges d'yvoire, ein Geschenk an den Herzog Berry von Louis de Vendôme. Über Elfenbein informieren KoECHLIN 1906 (in André Michels kunstgeschichtlicher Reihe), hier v.a S. 481-498 mit bibl. Nachweisen S. 505-507, KoECHLIN, Les ivoires gothiques français, 2 Bde. 1924, GRODECKI 1974 sowie GABORITCHOPIN 1978, S. 164-174) und GABORIT-CHOPIN 1994.

123 Zur Malerei i.e.S. "Paris 1300-1500« siehe u.a. STERLING 1987. Für den kurzen Überblick DurrieU, Paul: La peinture en France, in: MiCHEL, Histoire de l'Art, Bd. 3,1, 1907, S. 101-171 (hier v.a. S. 137-171 zur Regierungszeit Karls VI., dort wiederum zur Miniaturenmalerei S. 155-171. Das Januarbild wird behandelt S. 164, die "Très Riches Heures« und die Brüder Limburg S. 166-168).

124 Behandelt unten in Kap. C. II. 1., hierzu die Angaben unten S. 173 mit den entspr. Anm.

Behandelt ebd., hierzu die Angaben unten S. 174 mit den entspr. Anm.

127

Behandelt ebd., hierzu die Angaben unten S. 175 mit den entspr. Anm.

Behandelt ebd., hierzu die Angaben unten S. 177 in Anm. 276.

128 Behandelt ebd., hierzu die Angaben unten S. 169 mit den entspr. Anm.

129 Ein »Horn des Einhorns«, une corne d'une unicorne, toute entière wurde von Macé Heron, conseiller und trésorier général des Herzogs von Berry, diesem 1416 zum jour de l'an geschenkt, AN KK 258, fol. 194r, ed. GUIFFREY, Inventaires, Bd. 1, 1894, S. 303, Nr. 1138, siehe Anhang - Katalog/Jahreslisten, Nr. 1694. Zu den Exotika des Herzogs GuIfFreY, Inventaires, Bd. 1, 1894, S. CXXIIf. Siehe LABORDE, Glossaire, 1872: Stw. »Licorne«, S. 359-365.

130 Un annel ou il a une pierre, dont Joseph espousa Nostre Dame, den Berry 1406 von Antoinette de Beaufort, der Frau des Jean le Meingre, geschenkt bekam, AN KK 258, fol. 84v, ed. LEBEUF, Recueil, Bd. 2, 1738, S. 261f. und GUIFFREY, Inventaires, Bd. 1, 1894, S. 161, Nr. 600, vgl. SCHLOSSER 1908, S. 31. Siehe Anhang - Katalog/Jahreslisten, Nr. 1356.

131 GuIfFreY, Inventaires, Bd. 2, 1896, S, 339. Vgl. BNF ms.fr. 6747 (Comptes du duc de Berry, fol. 25r-34v.: Compte de Lomer le Bez [Ber?, J.H.], 1418 [Verkaufsliste pour le fait de nostre guerre [...] et non pour autre cause]; zur Ordonnanz vom 24. November 1417 fol. 31r-34v), ed. GUIFFREY, Inventaires, Bd. 2, 1896, S. 339-344 (vgl. ebd., Bd. 1, 1894, S. XIII). Siehe auch unten S. 78, Anm. 33. In dieser Liste standen auch zwei Hss.: un breviaire a l'usaige de Paris en deux volumes« und une tres belle bible en latin qui fait du 
mehr erhalten, zumal auch die sich wandelnde Mode, der sich ändernde Zeitgeschmack, zur nicht seltenen Umarbeitung von Gegenständen führte ${ }^{132}$. Eine große Ausnahme ist das sogenannte »Goldene Rößl«133 (siehe Abbildung 2), ein Geschenk der Königin Isabeau de Bavière an ihren Gatten zum

roy Robert. - Auch direkte Kriegseinwirkungen hatten die Bestände dezimiert wie bspw. die Zerstörung der Schlösser Bicêtre, Etampes und Dourdan 1411 im Zuge des Bürgerkriegs zwischen Armagnacs und Bourguignons.

132 Und so scheute sich auch der Herzog von Berry als Sammler nicht, Gegenstände, die ihm geschenkt worden waren, weiterzuverschenken, wie den bel et gros balay longuet im Wert von 18.000 éucs, an dessen Erwerb sich der König, verteilt auf die Neujahrstage 1402 bis 1404 , beteiligt hatte, und den er diesem dann 1416, in ein Kreuz gesetzt, zurückschenkte (oder schenken wollte), siehe Anhang - Katalog/Jahreslisten, Nr. 1292.

133 Notiert im Inventaire des meubles et joyaux du roi Charles VI, BNF ms.fr. 21446, fol. 24r-24v. »Le Rössel d'or d'Altoetting « als Gegenstand wissenschaftlicher Behandlung im 19. Jh.: LABARTE 1869 und LABARTE, Inventaire du mobilier de Charles V, 1879, S. XIXf., der das »Goldene Rößl« als erster anhand eines Inventars Karls VI. identifizierte, sowie GERSTNER 1853/1854 und VALLET DE VIRIVILLE, Documents relatifs aux joyaux de Charles VI, 1858, neben anderen, im 20. Jh. v.a. BEZOLD 1905 (ausführliche Beschreibung mit Abdruck der Quellen) und FraNkenBURger 1924, dann FiLLITZ 1963, S. 753f. Einen bis in die sechziger Jahre reichenden Literaturbericht zur Beschäftigung mit dieser Plastik bei STRAUB 1965, S. 199ff. Erwähnt unter kulturhistorischen Gesichtspunkten als ein Beispiel unter vielen zur "Kunst im Leben« auch bei HUIZINGA 1975, S. 373, der die Plastik etwas ungerecht urteilend nauf der Mitte zwischen edler Skulptur und den Prunkstücken festlichen Pomps« angesiedelt sieht und abschätzig bemerkt: "Wie peinlich berührt hier die an plumpen Prunk verschwendete vollendete Kunstfertigkeit unser Bedürfnis nach Schönheit«, dabei doch nur die Relativität von Geschmacksurteilen offenbart (kritisch über HUIZINGAs Urteil auch PANOFSKY, Early Netherlandish Painting, Bd. 1, 1953, S. 80; siehe MEUTHEN 1990, S. 108f. mit Anm. 61-64 zusammenfassend zu den kritischen Positionen). "Le petit cheval d'or « behandelt im Rahmen der Beschäftigung mit den königlichen Inventaren und der Verwaltung des königlichen Schatzes HENwOOD 1980, S. 194 mit Anm. 2 sowie S. 200 mit Anm. 2 (die profunde Arbeit STRAUB 1965 scheint HENWOOD zumindest in seinen Aufsätzen nicht rezipiert zu haben, seine Habilitationsschrift stand mir leider nicht zur Verfügung) und STRAUB 1996, S. 322. Das "Goldene Rößl« als kunsthistorisches Untersuchungsobjekt und Beleg für die hochentwickelte Pariser Goldemailtechnik bei MüLLER, STEINGRÄBER 1954, S. 39-43, 69-71, Nr. 6 (zuvor kurz behandelt bei EVANS 1948, S. 208 und PANOFSKY, Early Netherlandish Painting, Bd. 1, 1953, S. 69, 73, 80, 164 mit Tafel 13, Abb. 27, 28) und MIDDELDORF 1960, S. 237, fig. 2 und S. 239 mit 244, Anm. 66, sowie im Rahmen zahlreicher Überblickswerke wie STEINGRÄBER, Erich: Kunsthandwerk, in: BIALOSTOCKI 1972, S. 311-338, hier S. 312,318 mit Nr. LXIII, 289, GAUTHIER 1972, S. 298, 300, 416, Nr. 242 oder FRITZ 1982, S. 66, 107, LIGHTBOWN, Ronald W.: The migration period and the later middle ages, in: BLAIR 1987, S. 37-65, hier Abb. S. 64, und HABSBURG 1997, S. 12-15 mit Abb. 1 und 4. Auf hohem Niveau beschrieben und interpretiert bei EIKELMANN 1984, S. 210-229. Siehe auch HILGER 1992, S. 24f. mit Abb. 11 auf S. 25. Im aus Anlaß der gleichnamigen Ausstellung des Bayerischen Nationalmuseums in München vom 3. März bis zum 20. April 1995 herausgegebenen Ausstellungskatalog: Das Goldene Rössl. Ein Meisterwerk der Pariser Hofkunst um 1400, 1995, werden, vom konkreten Einzelfall ausgehend, in zahlreichen Einzelstudien sämtliche damit in Zusammenhang stehenden Aspekte beleuchtet. Siehe neuerdings auch GUENÉE 1999, S. 11-14 und STRATFORD, Rössl, 2000 (eine verb. Version von STRATFORD 1995). 
Neujahrstag $1405^{134}$, das, verpfändet an den Bruder Isabeaus, Ludwig den Bärtigen, die Zeitläufte einigermaßen unversehrt in der Kirche zu Altötting überdauert hat und nicht zuletzt aus eben diesem Grund die prominenteste Gabe der Zeit zum neuen Jahr überhaupt ist ${ }^{135}$.

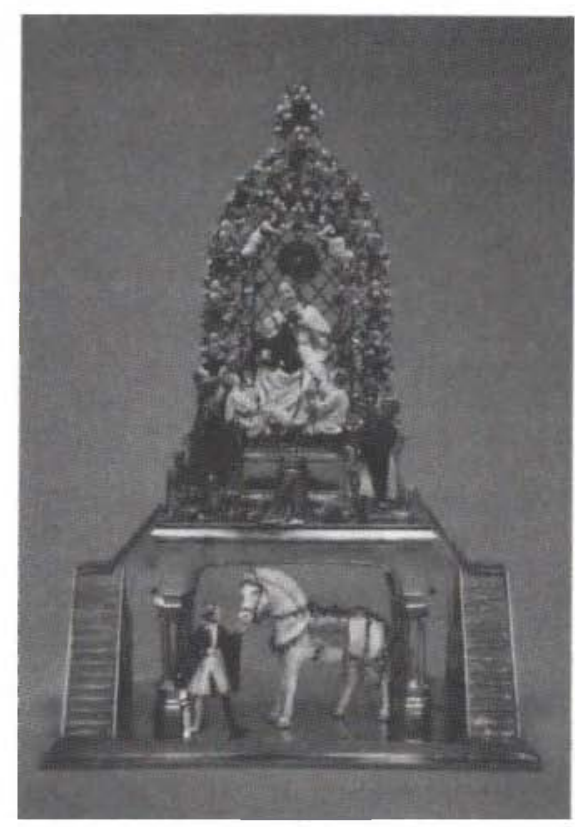

Abb. 2: Das Goldene Rößl, ein Neujahrsgeschenk der Königin

Isabeau für König Karl VI. 1405

Schatzkammer der Wallfahrtskirche Unserer Lieben Frau, Altötting

Cliche Bayerisches Nationalmuseum, München

Die entsprechende Notiz im "Inventaire des meubles et joyaux du roi Charles VI ${ }^{136}$ lautet: Item une imaige de Nostre Dame, qui tient son enfant assis en un jardin faict en maniere de traille, et est esmaillee ladicte Nostre Dame de blanc, et l'enfant de rouge cler, et a ladicte image un fermail en sa poictrine garny de six perles, et un balay, et audessus de la teste Nostre Dame a une couronne garnie de deux balasseaux, et un saphir et seize perles, et est

134 Die von David 1945, S. 33 und David 1947, S. 155 gegebene Datierung 1404 orientiert sich am alten Stil, ebenso bspw. BARRY 1964, S. 491 und KIMM 1969, S. 280f., aber auch HUIZINGA 1975, S. 373. Zum Jahresanfang siehe oben S. 51 mit den entspr. Anm. 135 Ausgezeichnete Behandlung des Schicksals dieses Kunstwerks aus der Perspektive Ludwigs des Bärtigen STRAUB 1965, S. 199ff. mit weiteren Nachweisen; vgl. STRAUB 1981, S. 155 mit Anm. 43.

136 BNF ms.fr. 21446, fol. 24r-24v, zitiert nach EIKELMANN, Geschichte des Marienbildes, 1995, S. 56. 
la tenue de ladicte couronne de deux angeloz esmaillez de blanc, et ledict jardin garny de cinq gros balaiz et cinq saphirs et trente deux perles, et a un lustrin ou il y a un livre dessus garny de douze perles, et au devant dudit image y a trois imaiges d'or, c'est assavoir sainte Catherine, saint JeanBaptiste, saint l'Evangeliste, et au dessoubz l'imaige du roy a genoulx sur un coussin garny de quatre perles, armé des armes de France et devant luy son livre sur un scabel d'or et derriere luy un tigre, et audevant du roy de l'autre costé a un chevalier armé et esmaillé de blanc et de bleu, qui tient le heaume du roy d'or, et au dessoubs en bas de l'entablement a un cheval esmaillé de blanc, et a la selle et le harnois d'or et un varlet emaillé de blanc et de bleu, qui le tient par une main par la bride et en l'autre main un baston, et poise environ dixhuict marcz d'or, et l'entablement sur quoy les choses dessus de sont ordonnees poise environ trente marcz d'argent doré, et fut donné par la Reine au Roy le premier jour de l'an 1404.

Eine Übersetzung gibt Renate Eikelmann ${ }^{137}$ : "Ein Bild Unserer Lieben Frau, die ihr Kind hält und in einem Garten sitzt, die in einer Art Laube gestaltet ist und diese Unsere Liebe Frau ist weiß emailliert und das Kind in Rubintorn, und dieses besagte Bild hat vor der Brust eine Agraffe, geschmückt mit sechs Perlen und einem Balas und oberhalb des Kopfes unserer Lieben Frau ist eine Krone, geschmückt mit zwei Balassen und einem Saphir und Perlen und diese besagte Krone wird von zwei weiß emaillierten Engeln gehalten, und der besagte Garten ist geschmückt mit fünf großen Balassen und fünf Saphiren und zweiunddreißig Perlen, und es gibt ein Pult mit einem Buch, welches oberhalb mit zwölf Perlen geschmückt ist und auf diesem Bild sind drei Bilder aus Gold, die hl. Katharina, hl. Johannes Baptista, hl. Johannes Evangelista und darunter ist das Bild des Königs, der auf einem Kissen kniet, geschmückt mit vier Perlen, geschmückt mit den Wappen von Frankreich. Vor ihm ein Gebetbuch auf einem goldenen Pult und hinter ihm ein Tiger und vor dem König auf der anderen Seite ist ein Ritter in Rüstung, emailliert in weiß und blau, der den Helm des Königs in Gold hält und unten auf dem Unterbau steht ein weißemailliertes Pferd mit einem Sattel und Zaumzeug aus Gold und ein Diener, weiß und blau emailliert, der es mit einer Hand am Zügel hält und in der anderen Hand einen Stock und es wiegt um 80 Mark an Gold und der Untersatz mit den unteren Dingen wiegt um dreißig Mark an vergoldetem Silber und es wurde von der Königin dem König am ersten Tag des Jahres 1404 geschenkt $\ll$.

Das festin du janvier markiert also im Rahmen des Geschenkverkehrs zum neuen Jahr kein singuläres Phänomen, sondern steht - selbst ein Höhepunkt künstlerischer Darstellung wie das 》Goldene Rößl« - symptomatischsignifikant für ein laut Überlieferung und Forschungsstand zuvor nicht 
erreichtes qualitatives und quantitatives Niveau höfischer Kunstproduktion. Dieses Niveau ist vor allem in der Kunstgeschichte seit langem in seiner Bedeutung bekannt und entsprechend ausgiebig behandelt worden ${ }^{138}$. Erich Müller und Theodor Steingräber verorteten dieses Phänomen in Zeit und Raum als die von ihnen noch so bezeichnete »französisch-burgundische« Goldemailkunst, die mit Renate Eikelmann treffender »franko-flämisch« zu nennen ist ${ }^{139}$, die den größten Teil der Neujahrsgeschenke ausmacht und als "Leitwährung" des Geschenkverkehrs gelten kann. Das Neuartige in der Darstellung seien »bildhafte Wirkungen von einer völlig neuen Unmittelbarkeit ${ }^{140}$, erzielt durch die neue Technik des émail en ronde bosse, die es erstmals ermöglichte, runde, meist aus Gold geformte Körper völlig mit Email zu überziehen, farbig zu gestalten und vielfältig zu verzieren ${ }^{141}$, in den Quellen bezeichnet als émaux peints, d'or émaillé, toute émaillé oder émaillé en blanc etc. Paris galt ihnen als Ausgangspunkt und wichtigstes Zentrum dieser Entwicklung, wobei bis 1420 das Gemeinsame überwogen habe und die Hofmode erst ab 1420 in lokale Besonderheiten zerfallen sei ${ }^{142}$. Renate Eikelmann hat diesen Befund in ihrer Dissertation über die »Franko-flämische Emailplastik« von 1984 auf der Grundlage miteinbezogener schriftlicher, vor allem archivalischer Quellen der Zeit um 1400 dahingehend modifiziert, daß neben Paris den anderen Fürstenhöfen in Bourges, in Angers und vor allem in Dijon und den niederländischen Kunstzentren gleichrangige Bedeutung zukomme, getragen von einer internationalen Künstlerschaft, wobei die Goldschmiedekunst in enge Beziehung zur zeitgenössischen Buchmalerei und Monumentalplastik zu setzen sei ${ }^{143}$. Das »Goldene Rößl « gehört dabei bis heute zweifelsfrei «zu den bedeutendsten erhaltenen Schöpfungen der Goldschmiedekunst am Hof Karls VI.«144 Tatsächlich waren neben den französischen

138 Aktuell im einschlägigen Zusammenhang BUETTNER, Brigitte: Past Presents: New Year's Gifts at the Valois Court, ca. 1400, in: The Art Bulletin 83, 2001, S. 598-624.

139 EIKELMANN 1984.

140 MÜLLER, STEINGRÄBER 1954, S. 29. So behandelt das »Goldene Rößl« ganz offenbar eine bis dahin in der künstlerischen Gestaltung nicht übliche Thematik: die spontane (?) Unterbrechung einer Reise, die König und Ritter für eine Andacht nutzen, die Darstellung also eines privaten Momentes, siehe KAHSNITZ 1995, S. 83f.

141 Zu dieser Technik EIKELMANN 1984, S. 18-121; MIDDELDORF 1960 in einem eigenen Aufsatz »On the Origins of `Email sur Ronde-Bosse« «. Bezogen auf Philipp den Kühnen hat LIGHTBOWN 1992, S. 161 festgestellt, daß die neue Technik dazu führte, daß der Herzog ab 1382 wand from this time onwards brooches with motifs enammelled in white regelmäßig kaufte.

142 Vgl. MÜLLER, STEINGRÄBER 1954, S. 32f. Differenziert EIKELMANN 1984, S. 14-17 und v.a. S. 513ff. Das Datum korrespondiert mit dem Einzug Heinrichs V. in Paris, vgl. auch BIALOSTOCKI 1972, S. 25.

143 EIKELMaNN 1984, S. 514f. - ebd., S. 122-175 zu den Zentren der Produktion von Goldschmiedearbeiten.

144 MÜller, STEINGRÄBER 1954, S. 39. Nach STRATFORD 1995, S. 46 muß das Goldene RößI, als Indikator für die sich steigernden Werte der von Isabeau verschenkten Neujahrs- 
Höfen Prag145 und Avignon weitere bedeutende Zentren des künstlerischen Schaffens, Avignon vor allem durch seine italienische Einflüsse nach Frankreich vermittelnde Rolle ${ }^{146}$. Paris verlor seine führende Stellung insbesondere durch die Katastrophe der verlorenen Schlacht von Azincourt 1415 und den Tod des Herzogs von Berry 1416 an die großen Städte der (nachmals) burgundischen Niederlande. Die stilbestimmten zeitlichen und räumlichen Grenzen setzt in diesem Sinn in kunsthistorischer Hinsicht der von etwa 1360 bis 1430 herrschende "Internationale Stil« der Spätgotik ${ }^{147}$. Dieser kunsthistorische Begriff verweist neben dem Vorhandensein eines winternationalen

geschenke, im Zusammenhang mit der sich ändernden politischen Bedeutung der französischen Königin gesehen werden, vgl. auch GIBBONS 2000, S. 392. Zum Kunsthandwerk der Zeit im Überblick STEINGRÄBER, Erich, in: BLALOSTOCKI 1972, S. 311-338; älter, aber grundlegend LABARTE, Histoire des arts industriels du moyen âge, 6 Bde., 1864-1866.

145 Siehe Die Parler und der Schöne Stil 1350-1400 (Ausstellungskatalog), 4 Bde., 19781980.

146 Siehe die zusammenfassenden Aussagen bei HILGER 1992, hier zum Zusammenhang der Pariser mit der Prager Kunst v.a. S. 12, 13ff., 17, zur Rolle Avignons S. 19. Freilich gab es noch eine ganze Reihe weiterer Berührungspunkte mit der Kunstszene Italiens, von denen die Heirat Ludwigs von Orléans mit Valentina Visconti nur einer war.

147 Nach der Datierung von VERDIER, Philippe: Introduction, in: The International Style. The Arts in Europe around 1400 (Ausstellungskatalog), 1962, S. X-XV, hier S. Xf., orientiert am Entstehungszeitpunkt des Stundenbuches der Jeanne d'Evreux und dem Auftreten des Jan van Eyck (HILGER 1992, S. 12 gibt als Eckdaten 1380-1430). Statt vieler Werke soll neben diesem Katalog und Europäische Kunst um 1400 (Ausstellungskatalog), 1962 (hier die Beiträge LHOTSKY, Alphons: Die Zeitenwende um das Jahr 1400, S. 5-26, SCHULTENORDHOLT, Hendrik: Die geistesgeschichtliche Situation der Zeit um 1400, S. 27-51, PÄCHT, Otto: Die Gotik der Zeit um 1400 als gesamteuropäische Kunstsprache, S. 52-65, sowie die einzelne Gegenstandsbereiche behandelnden Einführungen STERLING, Charles: Die Malerei um 1400, S. 66-78, PORCHER, Jean: Die Buchmalerei um 1400, S. 163-166, VERLET, Pierre: Die Goldschmiedekunst um 1400, hierzu die Nr. 124-129, und KING, Donald: Textilkunst um 1400, S. 435-441), nur noch auf Bd. 7 der Propyläen-Kunstgeschichte verwiesen werden, zitiert als BIALOSTOCKI 1972. Synonym verwendet für den Internationalen Stil werden auch die Termini »weicher Stil« und »schöner Stik, siehe zum Begriff im Überblick ebd., S. 25ff. Hans Peter HILGER hat in seinem kenntnisreichen Beitrag über »Aspekte der französischen Hofkunst um 1400« unter Verweis auf: Europäische Kunst um 1400 (Ausstellungskatalog), 1962, S. 11 (nach dem Beitrag LHOTSKY, Alphons: Die Zeitenwende um das Jahr 1400, S. 5-26) die nebeneinander existierenden Begriffe für den "Zeitstil 1400« noch einmal nach ihrer wissenschaftsgeschichtlichen Herkunft aufgeführt. So geht die Wendung "Internationaler Stil« zurück auf Louis COURAJOD (nicht zur Verfügung stand die Arbeit von Ulrike HEINRICH-SCHREIBER über »Die Bildhauerkunst in Paris zwischen 1360 und 1420. Vincennes und die französisch-höfische Skulptur unter Karl V. und Karl VI.«, Diss. Univ. Freiburg i. Br. 1972, die wahrscheinlich das Bild hätte abrunden können). Der sich v.a. an Bildnerei und Malerei orientierende Begriff »weicher Stil« ist eine Schöpfung von Wilhelm PINDER und der "schöne Stil« gilt hauptsächlich zur Kennzeichnung der Prager Kunstproduktion von etwa 1350 bis 1400 und ist ein Ergebnis der tschechischen Forschung, siehe HILGER 1992, S. 12, diesbezüglich leider ohne Einzelbelege. 
Bewußtseins « (verstärkt durch die Künstlerwanderungen ${ }^{148}$ ), kontrastiert von dem aufkeimenden Nationalgefühl vor allem in England und Frankreich im Zuge des Hundertjährigen Krieges ${ }^{149}$ auch auf den Bereich der mittelalterlichen Mobilität. Bei der Frage nach den Kosten dieser Mobilität will Gerd Althoff den Blick sauf jene immateriellen Bereiche der >Ars donandi<, in denen Ehren und Auszeichnungen, Hulderweise und anderes als symbolisches Kapital gehandelt wurden $\ll$, gerichtet sehen 150 .

Damit kann in einer ersten Annäherung das Phänomen des höfischen Geschenkverkehrs im spätmittelalterlichen Frankreich um 1400 am Beispiel der Neujahrsgeschenke idealtypisch beschrieben werden. Jeweils zum Jahresanfang fand in Form eines festlichen Banketts in einer der fürstlichen Residenzen der Austausch von offensichtlich überaus wertvollen Geschenken zwischen dem Fürsten, hohen Adligen und Geistlichen, Familienmitgliedern und Angehörigen des Hofstaates statt ${ }^{151}$. Dieser Vorgang kann anhand der vorge-

148 Siehe TroEsCHER 1953, exemplarisch BAUDOIN 1987: "Destinées itinérantes des grands imagiers de la fin du moyen âge «.

149 Vgl. etwa BEAUNE 1985 und v.a. KRYNEN 1981, hierzu die Rez. MÜLlER, Heribert: Königtum und Nationalgefühl in Frankreich um 1400, in: HJb 103 (1983) S. 131-145 mit zahlreichen weiterfuhrenden Literaturangaben und Hinweisen, zuletzt ZINGEL 1994.

150 ALTHOFF 1992, S. 104f. Vgl. auch unten S. 140, Anm. 19.

151 Siehe auch ParaviCINI, Peur, pratiques, intelligences, 1985, S. 193. - Zu den historiographischen und literarischen Zeugnissen sowie weiteren materiellen Hinterlassenschaften im vorliegenden Kap. die Abschn. III. 2. und III. 3. - Die Frage der Definition des grundlegenden Vorgangs scheint trotz spärlicher Hinweise in der nicht-archivalischen Überlieferung (siehe unten S. 91ff.) ihre Antwort im Ritual zu finden (mit und im Rahmen von zeremoniellen und repräsentativen Aspekten, siehe aus geschichtswissenschaftlicher Sicht PARAVICINI, Werner: Zeremoniell und Raum, in: Zeremoniell und Raum, hg. von DEMS., 1997, S. 11-36, hier S. 14; vgl. die Beschreibung höfischen Geschenkverkehrs in der Ottonenzeit als »rituelles Schenken« bei SCHELLER 1997), das soziologisch betrachtet gekennzeichnet ist durch eine festgelegte Folge von Handlungen (Person wird beschenkt, andere Person nimmt an und erwidert), die v.a. zwei Komponenten beinhalten: Kommunikation kulturellen Wissens (Vermittlung und Bestätigung anerkannter Werte) und zweckorientiertes sozial gebundenes Vorgehen (Aktualisierung dieser Werte). Eine Reduzierung von Ritualen auf ihre gleichwohl charakteristischen Merkmale Stereotypisierung und Wiederholung würde diese allerdings einiger ihrer gesellschaftlichen Funktionen berauben. Siehe FORSTER, Till: Art. »Ritual«, in: Wörterbuch der Soziologie, hg. von ENDRUWEIT, TROMMSDORF, Bd. 2, 1989, S. 546f., grundlegend TURNER 1989 (vgl. ROST, 1994, S. 28-31), knapp SOEFFNER 1991. In diesem Sinn auch BERKING 1996, S. 19: "Schenken [ist] eine rituelle Praxis, mittels derer der aktuelle Kurswert der jeweiligen Beziehung mitgeteilt und für eine gewisse Zeit auf Dauer gestellt werden mag « und ebd., S. 25 die Bemerkung, daß eben erst der rituelle Kontext die Kenntnisnahme gebietet. BERKING unterscheidet ebd., S. $31 \mathrm{f}$. zwischen »Zugangs-«, »Bestätigungs-« und »Gemeinsamkeitsritualen« und definiert letztere S. 32 durch »Geschenke anläßlich der Wiederkehr bestimmter, idiolatrischer Jahrestage, die der gemeinsamen Nutzung und der Gestaltung einer gemeinsamen Zukunft zugedacht sind [...] « Vgl. auch ALTHOFF 1990, S. 182ff. und allg. ALTHOFF 1993 zu den "Spielregeln der Kommunikation in mittelalterlicher Öffentlichkeit«. 
stellten Einzelfälle ikonographisch und archivalisch belegt werden ${ }^{152}$. Die vor allem auf chronikalisch-narrativen Quellen beruhende (kultur-) historische Forschung zu den großen Komplexen »Hof« und »höfische Kultur« hat die kulturgeschichtliche Bedeutung und Besonderheit der höfischen Kultur herausgearbeitet ${ }^{153}$. Kunsthistorische Erkenntnisse wiederum bestätigen der höfischen Kultur der Zeit das an ihrer Kunstproduktion ablesbare hohe Niveau.

Kulturgeschichtsforschung 154 und Kunstgeschichte haben also in nicht immer eindeutiger Trennung den Charakter der ins Auge gefaßten Epoche sowohl in ihrer Gegenständlichkeit als auch in den der höfischen Kultur verpflichteten Formen in qualitativer und quantitativer Hinsicht erkannt, dargestellt und analysiert. Der gegebene Abriß mit Blick auf das konkrete Thema sollte dies exemplarisch zeigen. Doch auch für wirtschafts- und sozialgeschichtliche Fragestellungen ${ }^{155}$ haben sich aus der archivalischen

152 Daß nicht nur gefeiert wurde am jour de l'an, schildert eine Begebenheit vom Neujahrstag 1405 im Rahmen der Querelle de Graville et de Boucicaut, wiedergegeben in Kap. 6 der "Chronique normande« (hierzu der Art. von Gillette TYL-LABORY, in: Dictionnaire des lettres françaises, 1996, S. 288-290): Si avint que la végille du jour de l'an en suivant, le dit Boursicaut soupoit en la ville, si s'en vint après souper monter sur sa mulle, son page après qui portoit son épée, et un vallet à pié qui portoit une torche et commenda à Dieu chelx ou il avoit souppé, en disant: "Or voit Gueratville chier; s'il ne m'encontre en ceste nuit, je le tiens pour parjure et que ses convenanz ne sont pas de gentille homme." Si ne savoit que à venir lui estoit! Si encontra son adversaire plus tost qu'il ne cuidoit au carrefourt d'une rue, et la fu assailly, et dit: "Ribaud, deffent-toy!" et fu rué jus de dessus sa mulle, et batu et rebatu, trayné par le millieu de la boc du regnel et out deux horions d'espée sur sa teste; encore y part; et le tallon coupé et une main afollée [...], Chronique normande, ed. VALLET DE VIRIVILLE, 1892, S. 329f.

153 Zur höfischen Kultur allg. NAUMANN, MÜLLER 1929; Otto CARTELLIERIs »kulturhistorische Bilder« über den Hof der Herzöge von Burgund: CARTELLIERI 1926; selbstverständlich der bereits erwähnte »Herbst des Mittelalters«: HuIZINGA 1975; Europas Fürstenhöfe, hg. von DICKENS, 1978 (hier v.a. die Beiträge von Arthur Geoffrey DiCKENS selbst über $»$ Die Höfe des Mittelalters und das Aufblühen der Kultur«, S. 8-31: DiCKENS 1978, und Charles Arthur John ARMSTRONG über »Das goldene Zeitalter von Burgund«, S. 54-75: ARMSTRONG 1978); Bryan HOLME über den Glanz höfischen Lebens im Mittelalter: HOLME 1987; Princes, Patronage, and the Nobility, hg. von ASCH, BIRKE, 1991 (hier v.a. der Beitrag PARAVICINI, The Court of the Dukes of Burgundy, 1991); Rainer A. MÜLlER über den Fürstenhof in der frühen Neuzeit: MÜLLER 1995; PARAVICINI 1994 (siehe oben S. 47, Anm. 57) und auch KRABS 1996 über Glanz und Elend der höfischen Welt.

154 Zur Einführung: HUIZINGA 1930; HASHAGEN 1950 (grundlegend); SCHWARZ 1970; LEGOFF 1970 und GUENÉE 1980. POIRIER, Histoire des moeurs, 3 Bde., 1990-1991. ist allg. zeitlich übergreifend und volkskundlichen Charakters, siehe hier v.a. die Beiträge MOLET, Louis: Comput et Calendriers, in: Bd. 1, 1990, S. 181-268 und BERNARD, Philippe J.: L'Homme et l'Economie, in: Bd. 2, 1991, S. 437-496, siehe S. 472-474 zu »don« und "contre-don«.

155 Einen ersten Überblick über die wirtschafts- und sozialgeschichtlichen Hintergründe der Zeit geben BULST, Neithart: Art. »Frankreich. C. Siedlungs-, Wirtschafts- und Sozialgeschichte «, in: LexMA, Bd. 4, 1989, Sp. 783-793, sowie Henri DuBOIS in seinem Beitrag "Frankreich 1350-1500 « für das Handbuch der europäischen Wirtschafts- und Sozialgeschichte (hg. von KELLENBENZ, 1986): DUBOIS 1986, alsdann neben HEERS 1973 mit Blick 
Überlieferung der Zeit Erkenntnisse ergeben, die beispielsweise über den Kreis der eigentlichen Produzenten der kunsthandwerklichen Erzeugnisse ${ }^{156}$ oder über das höfische Rechnungswesen und die beteiligten Ämter und Institutionen informieren und damit auch Antworten auf die Frage nach der Finanzierungsgrundlage einer teilweise derart aufwendigen Repräsentation, wie sie in den dazu verwendeten Gegenständen aufscheint, geben ${ }^{157}$.

auf Gesamteuropa: Europäische Wirtschaftsgeschichte, hg. von CIPOLLA, BORCHARDT, 1983. Neueren Datums ist ConTAMnNe, L'économie médiévale, 1993, Bibl. S. 413-424. Siehe auch SCHMIDTCHEN, Volker: Technik im Übergang vom Mittelalter zur Neuzeit 13501600, in: LUDWIG, SCHMIDTCHEN 1992, S. 209-598, Bibl. S. 607-621.

156 Stellvertretend genannt sei die Arbeit Philippe HENwOODs über die Pariser Goldschmiede 1380-1422: HENWOOD 1982. - Zu Teilbereichen: Artistes, artisans et production artistique au moyen âge, hg. von ALTET, 3 Bde., 1986-1990.

$157 \mathrm{Zu}$ Johann von Berry sind diesbezüglich heranzuziehen neben LEHOUX, Jean de France, 3 Bde., 1966-1968 und AUTRAND, Jean de Berry, 2000 u.a. LACOUR 1934 und REY, Les Finances Royales sous Charles VI, 1965, hier S. 593ff., vgl. FAVIER 1966. - Siehe auch die entspr. Ausführungen im vorliegenden Kap. in Abschn. III. 1. 